\title{
Glycans pattern the phase behaviour of lipid membranes
}

\section{Citation}

Subramaniam, Anand Bala, Guido Guidotti, Vinothan N. Manoharan, and Howard A. Stone. 2012. Glycans Pattern the Phase Behaviour of Lipid Membranes. Nature Materials 12, no. 2: 128-133. doi:10.1038/nmat3492.

\section{Published Version}

10.1038/nmat3492

\section{Permanent link}

http://nrs.harvard.edu/urn-3:HUL.InstRepos:29996532

\section{Terms of Use}

This article was downloaded from Harvard University's DASH repository, and is made available under the terms and conditions applicable to Open Access Policy Articles, as set forth at http:// nrs.harvard.edu/urn-3:HUL.InstRepos:dash.current.terms-of-use\#OAP

\section{Share Your Story}

The Harvard community has made this article openly available.

Please share how this access benefits you. Submit a story.

Accessibility 


\title{
Glycan networks pattern the phase behaviour of lipid membranes
}

Authors: Anand Bala Subramaniam ${ }^{1, *}$, , Guido Guidotti ${ }^{2}$, Vinothan N. Manoharan ${ }^{1}$, Howard A. Stone ${ }^{3, *}$

\begin{abstract}
Affiliations:
${ }^{1}$ School of Engineering and Applied Sciences, Harvard University, Cambridge MA 02138.

${ }^{2}$ Department of Molecular and Cellular Biology, Harvard University, Cambridge MA 02138.

${ }^{3}$ Department of Mechanical and Aerospace Engineering, Princeton University, Princeton NJ 08544.

*Correspondence to: balasubr@fas.harvard.edu, hastone@ princeton.edu.

$\dagger$ Current Affiliation: Department of Chemistry and Chemical Biology, Harvard University, Cambridge MA 02138.
\end{abstract}


Hydrated networks of glycans (polysaccharides) - in the form of cell walls, periplasms or gel-like matrices - are ubiquitously present adjacent to cellular plasma membranes ${ }^{1-4}$. Yet despite their abundance, the function of glycans in the extracellular milieu is largely unknown ${ }^{\mathbf{5}}$. Here we show that the spatial configuration of glycans controls the phase behaviour of multiphase model lipid membranes: inhomogeneous glycan networks stabilize large lipid domains at the characteristic length scale of the network, whereas homogeneous networks suppress macroscopic lipid phase separation. We also find that glycan-patterned phase separation is thermally reversible - thus indicating that the effect is thermodynamic rather than kinetic - and that phase patterning results likely due to a preferential interaction of glycans with ordered lipid phases. These findings have implications for membrane-mediated transport processes ${ }^{6-}$ ${ }^{8}$, potentially rationalize long-standing observations that differentiate the behaviour of native and model membranes ${ }^{9-13}$, and may indicate a more intimate coupling between cellular lipidomes and glycomes than realized currently.

Glycan-rich cell walls or extracellular matrices that are rigid in comparison to the plasma membrane surround most cells ${ }^{1-4}$. However, it is often reported that rigid supports cause non- 'equilibrium,14-16 behaviour of lipids and proteins in model lipid membranes. Indeed, while there are many reports of lipid membranes supported on non-biological polymers such as polyethylene glycol, polyacrylamide or polyethyleneimine ${ }^{17-20}$ which seek to mitigate non'equilibrium' behaviour, such systems have been used mainly to study the effects of polymer hydration ${ }^{21}$ on the mobility of lipids ${ }^{18,19}$, or to preserve the function of transmembrane protein inclusions ${ }^{17,22}$. Furthermore, while it is widely acknowledged that a greater understanding of parameters that influence the size and length-scale of membrane domains is required ${ }^{6-13,23,24}$, as far as we know, systematic studies exploring the effects of biopolymers (the subject of this letter), or other more commonly used polymers in the field ${ }^{17-20}$ on the phase behaviour of lipid membranes have not been conducted. To investigate the possible effects of biologically relevant polymers on the behaviour of membranes, we designed an in vitro solid-supported experimental platform that allows, through fluorescent labelling and confocal microscopy, the study of lipid membranes interacting with hydrated networks of glycans with arbitrary glycan composition and variable network configurations.

We prepare glycan networks on flat hydrophilic surfaces using instability driven pattern formation (Supplementary Information (SI) for full details). Spontaneous rupture of giant lipid vesicles provided 2D lipid membrane patches that interact with the glycan networks. Lipid membranes were labelled 
with trace amounts of fluorescent probes to visualize phase behaviour. Vesicle rupture was performed at $65^{\circ} \mathrm{C}$ to ensure that the membranes, which consisted of up to five distinct lipid species, were fully mixed in a single macroscopically uniform liquid phase ${ }^{11,13}$. The glycan network-lipid membrane system was then cooled to room temperature for subsequent experiments. While the lateral configuration of the glycan network does not change with temperature (Supplementary Figure 10), as we outline further below, the lipid membrane is free to undergo temperature induced phase transitions. Note further that while the images shown in this report are of FITC-labelled glucomannan, we find that all of the glycans tested have the same effects on our membranes, and thus we refer generically to glycans in the remainder of this report.

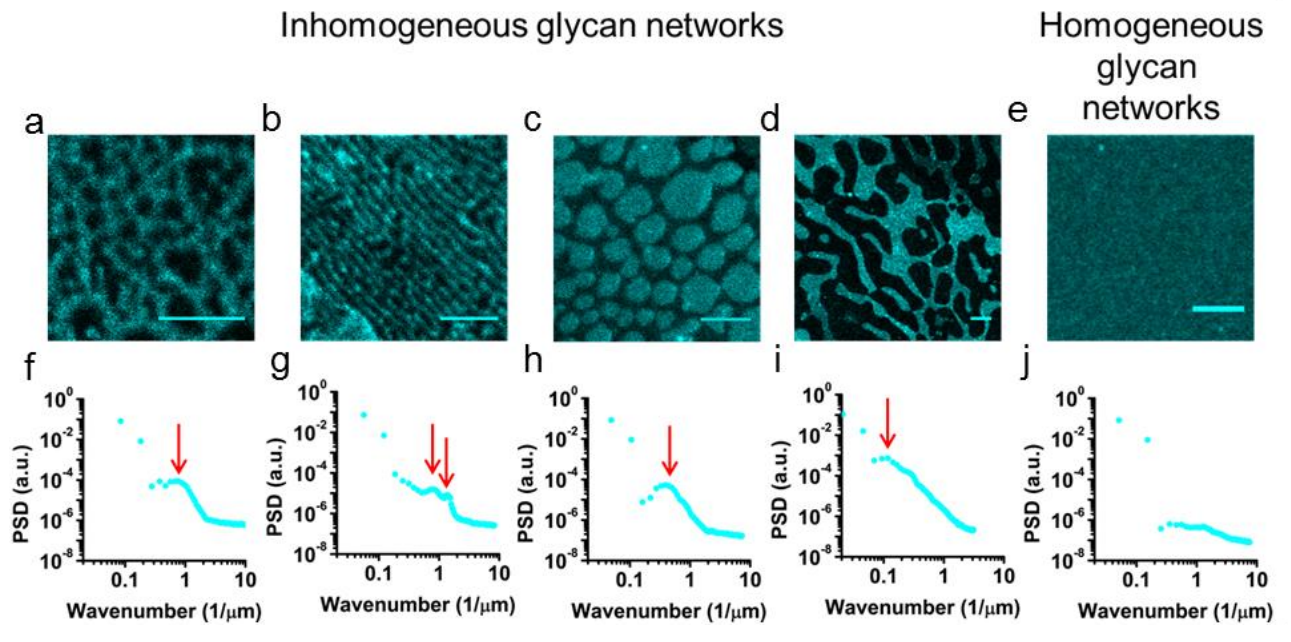

Figure 1 | Preparation of hydrated glycan networks with varying spatial configurations. (a-d) Inhomogeneous glycan networks are characterized by the presence of optically resolvable glycan-rich and glycan-poor domains. The shape and interdomain spacing varies with preparation conditions. (f-i) The power spectral density (PSD) of these networks reveals a pronounced peak (red arrow), at a wavenumber, sometimes two, signifying the dominant length scales present in the image. We call the reciprocal of these wavenumbers the characteristic length scale(s) of the network, $L_{\text {network }}$. For the sample networks shown here, $L_{\text {network }}=$ (f) $1.31 \mu \mathrm{m}$, (g) first peak, $1.31 \mu \mathrm{m}$, second peak, $0.74 \mu \mathrm{m}$, (h) $2.58 \mu \mathrm{m}$, (i) $8.50 \mu \mathrm{m}$. (e) Homogeneous glycan networks appear uniform with no resolvable glycan domains (j). There are no pronounced peaks in the PSD, signifying the absence of a dominant length scale, at least down to optical resolution ( $300 \mathrm{~nm}$ in our setup). Note also the distinct difference in the shape of the PSD curves of inhomogeneous (f-g) and homogeneous networks (j). Scale bars $5 \mu \mathrm{m}$. 
We prepare two classes of glycan networks: (i) inhomogeneous glycan networks, characterized by the presence of distinct glycan-rich and glycanpoor domains (Figure 1a-d), and (ii) homogeneous glycan networks, characterized by a uniform distribution of glycans with any inhomogeneities below the resolution limit of the microscope $(\sim 300 \mathrm{~nm})$ (Fig. 1e). To obtain a quantitative description of the network structure, we calculate the power spectral density (PSD) of the florescence intensity. For inhomogeneous networks, pronounced peaks (red arrows), typically one but sometimes two, can be discerned in the PSD curve at characteristic wavenumbers (Fig. 1f-i). We term the reciprocal of these wavenumbers as the characteristic length scale of the network, $L_{\text {network}}$. For homogenous networks, as expected, no pronounced peaks are present in the PSD curve, which confirms the lack of resolvable structures (Fig. 1j).

We find that multiphase membranes that rest on inhomogeneous glycan networks exhibit macroscopic time-stable lipid domains at room temperature (Figure 2 a,b). Membranes in a single phase on the other hand are insensitive to the glycan network (Supplementary Figure 1). Liquid ordered $\left(\mathrm{L}_{\mathrm{o}}\right)$ and solid ordered $\left(\mathrm{S}_{\mathrm{o}}\right)$ domains coexisting with liquid disordered $\left(\mathrm{L}_{\mathrm{d}}\right)$ domains on the glycan networks appear identical in shape, though other physical properties such as lipid diffusion coefficients vary as expected based on the known physical properties of these phases (SI, Supplementary Figure 4, 5). The lipid domains as a whole do not exhibit Brownian motion, have non-fluctuating boundaries, and do not coarsen over a two-day period, which differentiates their dynamics from multiphase lipid vesicles ${ }^{11,25}$ and from solid-supported double bilayers ${ }^{15,26,27}$. Fourier analysis of the fluorescence intensity of the lipid phase-sensitive probes allows us to determine the characteristic length scale of the phase-separated domains, $L_{\text {lipid }}$ : we find that $L_{\text {lipid }}$ (green arrows) matches $L_{\text {network }}$ (red arrows) (Fig. 2c,d). Indeed, $L_{\text {lipid }}$ is almost perfectly correlated with $L_{\text {network }}$ in multiphase membranes resting on inhomogeneous glycan networks (linear correlation coefficient $0.9975, n=15 \mathrm{~L}_{0}-\mathrm{L}_{\mathrm{d}}, n=15, \mathrm{~S}_{0^{-}}$ $\mathrm{L}_{\mathrm{d}}$; Fig. 2e). Thus, we conclude that the glycans in the network sets the characteristic length scale of phase separation of the adjacent multiphase membrane, Llipid. 


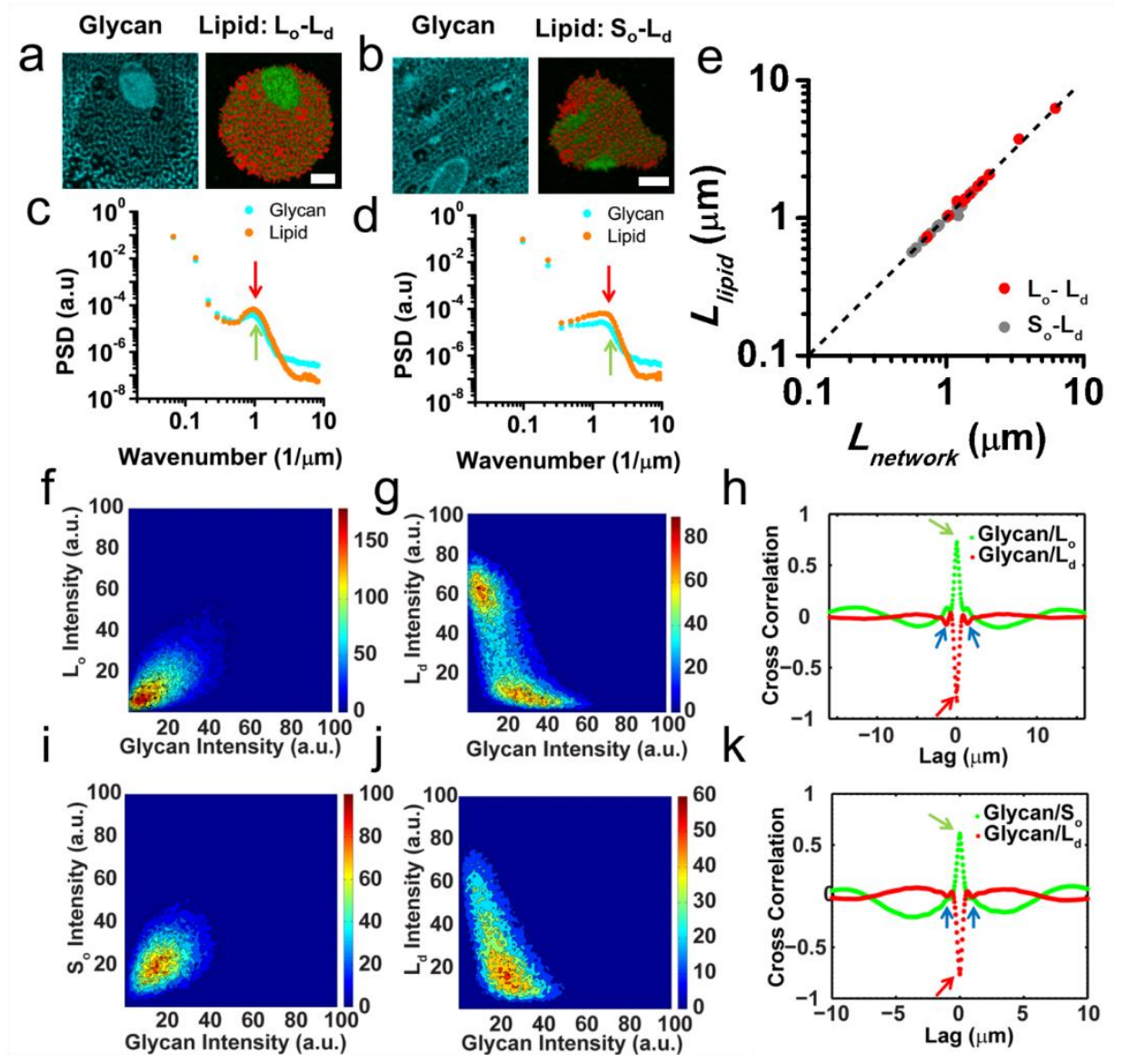

Figure 2 | Inhomogeneous glycan networks pattern the formation of macroscopic timestable lipid domains in multiphase membranes. $(\mathbf{a}, \mathbf{b})$ Inhomogeneous glycan networks pattern the formation of phase-separated lipid domains in multiphase membranes showing (a) $\mathrm{L}_{\mathrm{o}}-\mathrm{L}_{\mathrm{d}}$ and (b) $\mathrm{S}_{\mathrm{o}}-\mathrm{L}_{\mathrm{d}}$ coexistence. (c,d) PSDs show clearly that $L_{\text {lipid }}$ (red arrows) matches $L_{n e t w o r k}$ (green arrows). (e) Plot of $L_{\text {network }}$ versus $L_{\text {lipid, }}$ identified from the PSD of $n=15$ membranes in the $\mathrm{L}_{0}-\mathrm{L}_{\mathrm{d}}$ phase and $n=15$ membranes in $\mathrm{S}_{\mathrm{o}}-\mathrm{L}_{\mathrm{d}}$ phase. The diagonal line is a guide to the eye showing the case for perfect correlation. There is strong correlation between $L_{\text {network }}$, and $L_{\text {lipid; }}$; the linear correlation coefficient, $r=0.9975$. (f) Contour plots of the grayscale intensity of $\mathrm{L}_{\mathrm{o}}$ preferring probes vs. glycan shows a high density of points along the diagonal indicative of colocalization of $\mathrm{L}_{\mathrm{o}}$ domains and glycans. (g) For $\mathrm{L}_{\mathrm{d}}$ sensitive probes, a high density of points along the two axes and away from the diagonal indicates anticorrelation of $\mathrm{L}_{\mathrm{d}}$ domains and glycans. (h) Radial average of the normalized cross-correlation matrix of the glycan network and $\mathrm{L}_{\mathrm{o}}$ domains (green filled circles) and the glycan network and $\mathrm{L}_{\mathrm{d}}$ domains (red filled circles). The large positive peak at zero lag (green arrow) shows that $\mathrm{L}_{\mathrm{o}}$ domains are positively correlated with the glycans in the network, while the large negative peak at zero lag (red arrow) shows that $\mathrm{L}_{\mathrm{d}}$ domains have a negative correlation with the distribution of glycans in the network. Secondary peaks (blue arrows) show the crosscorrelation lengths. (i-k) Similar analysis performed on the $\mathrm{S}_{\mathrm{o}}-\mathrm{L}_{\mathrm{d}}$ membrane shown in (b) shows that $\mathrm{S}_{\mathrm{o}}$ domains also rest on glycan-rich regions. Note that the close proximity of the membrane to the glycan layer results in the quenching of fluorescence intensity of the glycan due to FRET between rhodamine in the $\mathrm{L}_{\mathrm{d}}$ probes and FITC in the glycan (See SI for further details). (a-b) Scale bars $5 \mu \mathrm{m}$. 
How do the glycans set $L_{\text {lipid }}$ ? We use two methods to analyse the positions of lipid domains with respect to the glycan network. First, we compare the greyscale intensity of $L_{d}$ and $L_{o}$ pixels against the grey-scale intensity of glycan pixels and plot the resulting datasets as color-coded contour plots. For $\mathrm{L}_{\mathrm{o}}$ sensitive probes, a high density of points lies along the diagonal, which demonstrates the colocalization of $\mathrm{L}_{0}$ preferring probes and glycans (Fig. 2f). In contrast, for $\mathrm{L}_{\mathrm{d}}$ sensitive probes a high density of points along the two axes and away from the diagonal indicates anti-correlation of $\mathrm{L}_{\mathrm{d}}$ preferring probes and glycans (Fig. 2g, also see Supplementary Figure 9 for multi-image analysis). Second, we cross-correlate glycan images with $\mathrm{L}_{\mathrm{d}}$ and $\mathrm{L}_{\mathrm{o}}$ images and calculate the radial average of the resulting 2D cross correlation matrices for $n=15 \mathrm{~L}_{\mathrm{o}}-\mathrm{L}_{\mathrm{d}}$ bilayers. We show the cross-correlation curves corresponding to the images in Fig. 2a as Fig. 2h. We find that $\mathrm{L}_{\mathrm{o}} /$ glycan cross-correlation curves show a large positive peak at zero lag (amplitude $=0.72$ for curve in Fig. $2 \mathrm{~h}, 0.63 \pm 0.09$ for $n=15 \mathrm{Lo} /$ glycan images), which indicates that the locations of $\mathrm{L}_{\mathrm{o}}$ domains are positively correlated with those of glycan-rich domains. On the other hand, $\mathrm{L}_{\mathrm{d}} / \mathrm{glycan}$ cross-correlation curves show a large negative peak at zero lag (amplitude $=-0.83$ for curve in Fig. $2 \mathrm{~h},-0.72 \pm 0.09$ for $n=15 \mathrm{Ld}_{\mathrm{d}}$ glycan images), which indicates that the position of $\mathrm{L}_{\mathrm{d}}$ domains is negatively correlated with the position of glycan-rich domains. The crosscorrelation length $\sim 1 \mu \mathrm{m}$ (blue arrows), is in good agreement with $L_{\text {network }}$ found from the PSD in Fig. 2c. Similar analyses performed on $S_{0}-L_{d}$ membranes indicate that $S_{o}$ domains colocalize with glycan-rich regions (Fig. $2 \mathrm{i}-\mathrm{k}$ ) (mean peak amplitude $=0.61 \pm 0.17$ for $n=15 \mathrm{~S} /$ glycan images, $=$ $0.77 \pm 0.13$ for $n=15 \mathrm{~L}_{\mathrm{d}} /$ glycan images ${ }^{18}$ ). We thus conclude that the more ordered $\mathrm{L}_{\mathrm{o}}$ and $\mathrm{S}_{\mathrm{o}}$ phases of multiphase membranes have an affinity for glycan-rich regions. This affinity dictates the patterning of the characteristic length scale of the lipids, $L_{\text {lipid, }}$, at the characteristic length scale of the network Lnetwork.

The irregular shapes of $\mathrm{L}_{0}$ and $\mathrm{L}_{\mathrm{d}}$ domains are noteworthy since these phases in the absence of glycans are strongly influenced by line tension and thus minimize boundary perimeters by forming a small number of large circular domains ${ }^{12}$. Our sample of $15 \mathrm{~L}_{0}-\mathrm{L}_{\mathrm{d}}$ membranes, present excess perimeter boundaries ranging from 600 to $1200 \%$ of the theoretical minimum extrapolated from the domain areas. One possible explanation for the excess boundaries is kinetic trapping ${ }^{14}$. If this were the case, we would expect the membranes to not undergo reversible temperature-induced phase transitions $11,13,14,28$. We test for kinetic trapping by subjecting the membranes to thermal cycles. We find that lipid membranes on inhomogeneous glycan networks clearly maintain thermodynamic transitions: domains melt above the membrane transition temperature and reform below the transition temperature (Figure 3). Llipid however remains the same after the thermal cycle (Fig. 3). We 
conclude that the lipid domains are not trapped and that we are observing the inhomogeneous glycan network strongly modifying the characteristic length scale of phase separation, $L_{\text {lipid }}$. While the phase behaviour of membranes with kinetically trapped domains is subject to several subtleties such as the incomplete dissolution of domains when temperature is cycled ${ }^{26}$, it is important to note, however, that unlike in previous reports ${ }^{11,13,14,28,26}$, we ensured that our vesicles were in a single uniform phase upon initial fusion onto the glycan networks.

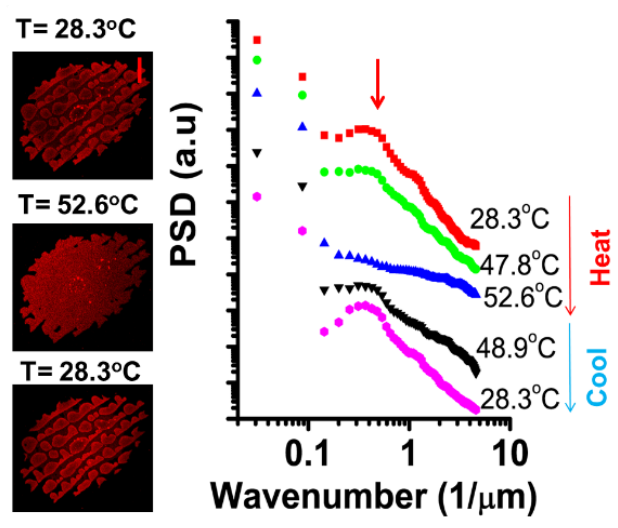

Figure 3 | Lipid domains on inhomogeneous glycan networks are in thermal equilibrium as evidenced by reversible temperature induced phase transitions. When heated, lipid domains melt, and the fluorescent phase sensitive probes distribute uniformly in the lipid membrane. When the membrane is cooled the domains reform. The peak in the PSD curve (red arrow) confirms that $L_{\text {lipid }}$ is preserved at the end of the thermal cycle. The PSD curves have been offset for clarity and the membrane is resting on an unlabelled hyaluronic acid network. The duration of the cycle is 10 minutes. Scale bar $15 \mu \mathrm{m}$.

How do multiphase membranes behave on homogenous glycan networks, where there is no discernible $L_{\text {network}}$ ? We find that multiphase membranes that rest on homogenous glycan networks appear uniform (Figure 4 a,b). Fourier analysis confirms that there is no characteristic length scale of phase separation, $L_{\text {lipid }}($ Fig. 4 c,d). Furthermore, intensity contour plots show a clustering of points in intensity phase space of $\mathrm{L}_{\mathrm{o}}, \mathrm{S}_{\mathrm{o}}$ and $\mathrm{L}_{\mathrm{d}}$ sensitive probes (Fig. 4 e,f,h,i) and cross-correlation curves are flat (Fig. 4 g,j). Indeed, peak amplitudes at zero lag for these membranes are an order of magnitude smaller in comparison to those on inhomogeneous glycan networks (values = $0.05 \pm 0.09$ and $0.07 \pm 0.14$ for $n=15 \mathrm{Lo} /$ glycan, $\mathrm{L}_{\mathrm{d}} /$ glycan images respectively, and $=-0.01 \pm 0.03$ and $-0.02 \pm 0.10$ for $n=15 \mathrm{~S}$ /glycan, $\mathrm{L}_{\mathrm{d}} /$ glycan images respectively). These results are consistent with the conclusion that there is no macroscopic phase separation of the lipids in the membranes, and contrasts sharply with the behaviour of lipids in similar membranes on inhomogeneous glycan networks (Fig. 2a,b) and in giant vesicles (Fig. S2d,e). We can neither support nor exclude the possibility that nanoscale domains or short-lived 
'rafts' with length scales below the resolution limit of the microscope ${ }^{29}$ may be present in multiphase membranes on homogeneous glycan networks.
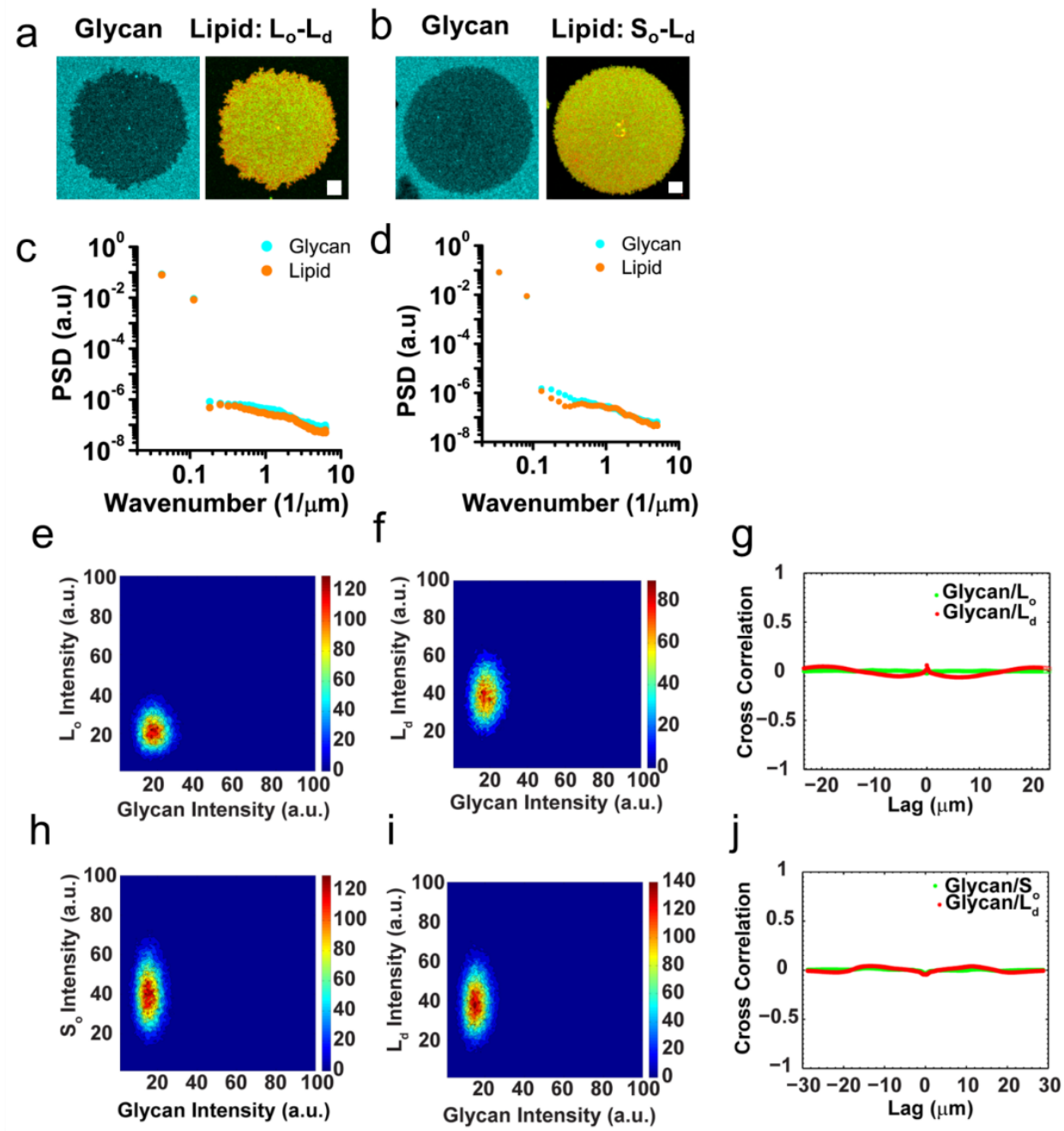

Figure 4 | Homogeneous glycan networks suppress phase separation in multiphase lipid membranes. (a,b) Multiphase membranes on homogenous glycan networks appear uniform. (c,d) PSD curves confirm the lack of a characteristic length scale in the membrane (orange circles). Glycan PSDs are shown as cyan circles (e,f) Contour plots of the grey-scale intensity of $\mathrm{L}_{\mathrm{o}}$ preferring probes vs. glycan and $\mathrm{L}_{d}$ sensitive probes vs. glycans show a cluster of points in intensity phase space. (g) Radial averages of the normalized cross-correlation matrices of the glycan network and $\mathrm{L}_{\mathrm{o}}$ image (green filled circles) and the glycan network and $\mathrm{L}_{\mathrm{d}}$ image (red filled circles). No clear correlations are evident. (h-j) Analysis performed on $\mathrm{S}_{\mathrm{o}}-\mathrm{L}_{\mathrm{d}}$ membrane shown in (b) gives a similar result. The results of both methods of analysis are consistent with the conclusion that there is no macroscopic phase separation of multiphase lipid membranes on homogeneous networks. Note that the close proximity of the membrane to the glycan layer results in the quenching of fluorescence intensity of the glycan due to FRET between rhodamine in the $\mathrm{L}_{\mathrm{d}}$ probes and FITC in the glycan (See SI for further details). Scale bars $5 \mu \mathrm{m}$. 
Differences in roughness ${ }^{24}$ might be a starting point for a mechanistic explanation of glycans effects on lipid membranes. We image our glycan networks and bare oxidized PDMS with an atomic force microscope (AFM). Typical images of the inhomogeneous and homogeneous networks are shown in Figure 5a,b. AFM phase imaging indicates that the number of glycan molecules per unit area is lower in the glycan poor regions than in the glycan rich regions (Fig. 5c, also see SI Section 6). We calculate the root mean square roughness ( $\mathrm{R}_{\mathrm{RMS}}$ ) values over a 1 x 1 micron square region to allow comparison of the roughness values between inhomogeneous glycan networks (we placed the sampling box over glycan-rich domains and glycan-poor domains of sufficient size to fully enclose the $1 \times 1$ micron box). For homogenous networks where there are no domains and for bare PDMS, the sampling box was placed at random. We found that the $\mathrm{R}_{\mathrm{RMS}}$ between the glycan-rich and glycan-poor regions were similar within experimental error (Fig. 5d). R RMS of the homogeneous glycan networks were also indistinguishable within experimental error, while bare oxidized PDMS had a lower R RMS (Fig. 5e).

The glycan-rich regions are raised with respect to the glycan-poor regions (Fig. 5a). Naively, gross changes in topography might be an alternate explanation for our observation of phase patterning in multiphase membranes. How do we untangle putative gross topographical effects from other chemical or microstructural features of the glycan network? We devised a method to test for the effects of topography by making replicas ${ }^{27}$ of the glycan network in PDMS. These replicas preserve the gross topography of the network while being composed purely of inert PDMS.

We deposit multiphase membranes on the PDMS replicas and visualize with the confocal microscope (Fig. 5f,g). We use confocal reflection microscopy to visualize the replica, since it is not fluorescent. Thus gray-scale intensity of the images of the replica corresponds to the height and curvature of the surface, and not the number density of glycans on the surface (which is what is measured in the fluorescence images). We perform Fourier analysis, similar to the ones performed for the glycan networks, on these images. It is clear from the images, the PSDs, and the cross-correlation analysis that the replicas do not pattern the phase separation of the membranes (Fig. 5h-k). It might be possible that there are differences at the nanoscale regarding the contours of the lipid bilayers and the substrates, but it is unlikely to be a main factor given the many reports that lipid bilayers follow faithfully the topography of even highly rough silica substrates ${ }^{15,24}$ (SI Section 7). 

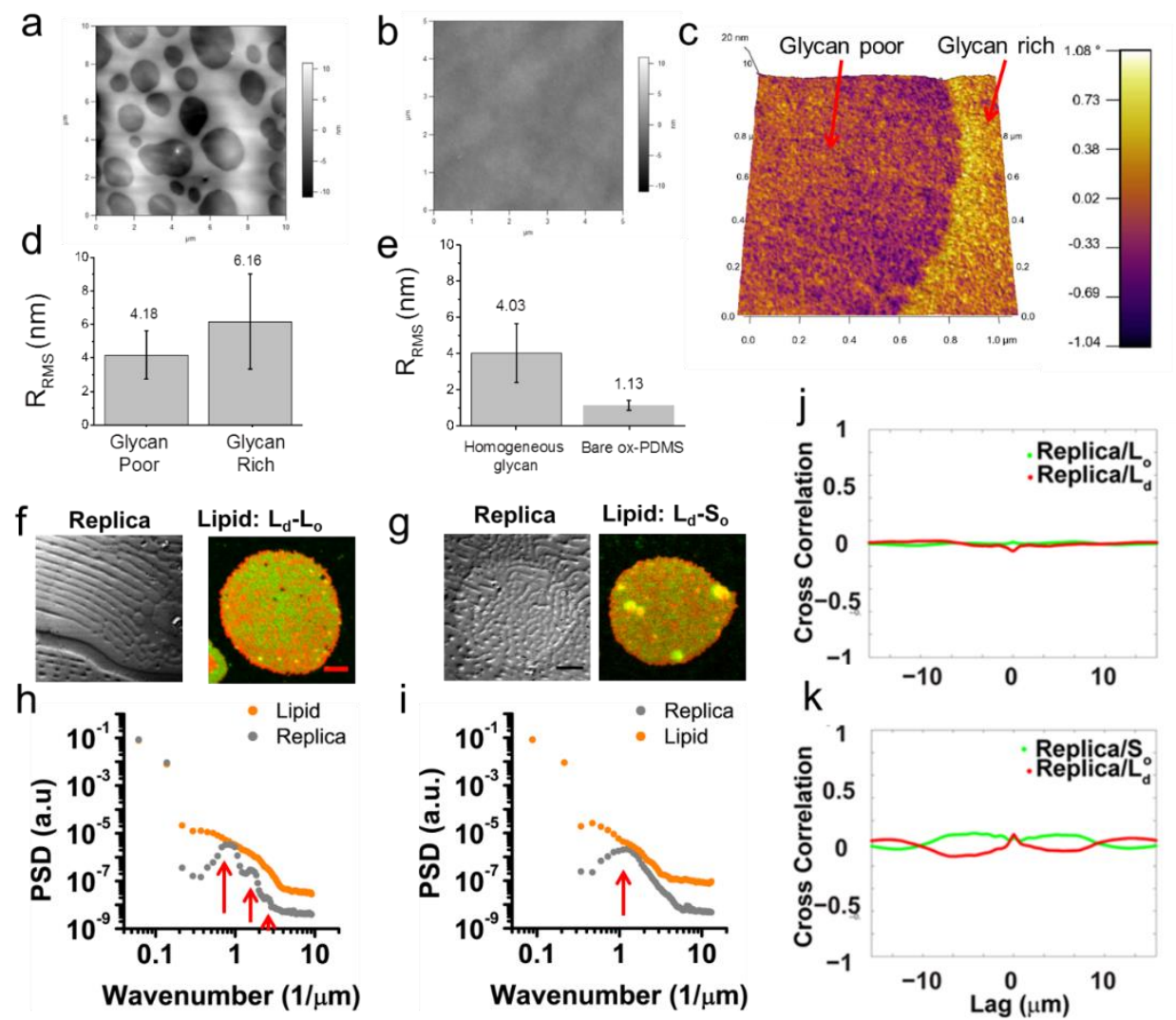

Figure 5 | Gross topography and microstructural roughness of the inhomogeneous glycan networks are not the dominant mechanism for phase patterning of lipid membranes. (a,b) AFM images of typical inhomogeneous and homogeneous glycan networks. The dark gray regions are glycan-poor domains and the light gray regions are glycan-rich domains. (c) A 3D reconstruction of the inhomogeneous glycan network overlaid with AFM phase information. The phase image was false colored according to phase angle, with glycans appearing yellow while the substrate appears purple. It is clear that glycans are present at a lower density in the glycan poor regions. (d) $\mathrm{R}_{\mathrm{RMS}}$ values indicate no significant difference in roughness between the glycan-rich and glycan-poor regions. (e) $R_{\text {RMS }}$ of homogeneous networks was comparable to those measured on inhomogeneous networks. Bare ox-PDMS (i.e. substrate devoid of glycans) was significantly smoother. Error bars are standard deviations for $n=10$. (f,g) Multiphase membranes deposited on the PDMS replicas under similar conditions as membranes shown in Fig. 2 and 4. The replica surface, which is not fluorescent, was imaged through confocal reflection microscopy (glycans cannot be imaged through reflection, likely because the highly hydrated layers lack optical contrast with the surrounding water). Bright yellow circles on the membrane are lipid vesicles adhering to the bilayer patch. Scale bars $5 \mu \mathrm{m}$. (h,i) PSDs of the bilayer (filled orange circles) show that the lipids do not respond to the dominant length scale of the PDMS replica (filled gray circles). (j,k) Radial averages of the normalized cross-correlation matrices of the replica and $\mathrm{L}_{\mathrm{o}} / \mathrm{S}_{\mathrm{o}}$ images (green filled circles) and the replica and $\mathrm{L}_{\mathrm{d}}$ images (red filled circles). No clear correlations are evident. 
Our experimental results are reproducible for (i) all the glycans tested: hyaluronic acid, heparan sulfate, pectin, inulin, carboxymethyl cellulose, glucomannan, dextran, and Ficoll, (ii) varying lipid species within the coexistence regimes such as DPPC, BSM, POPC, and ergosterol, and (iii) varying fluorescent-probe molecules. Furthermore, extensive control experiments were performed to explore potential fluorescent probe-related artefacts.

The effect of glycans that we report here could rationalize the discrepancy between previous observations of model lipid membranes, which were without glycans, and the various observations of domains, or lack thereof, in plasma membranes. The plasma membrane of mammalian cells for which nanodomains have been inferred ${ }^{6,9}$ are surrounded by gel-like extracellular matrices ${ }^{1}$ or glycan coatings ${ }^{2}$. The absence of macroscopic phase separation of these membranes matches the behaviour of lipid membranes adjacent to homogeneous glycan networks. The lipid domains on inhomogeneous glycan networks on the other hand resemble the large stable 'rafts' observed in yeast plasma membranes ${ }^{30,31}$ that are adjacent to a multilayered cell wall. We propose that that glycans could pattern the phase separation of membranes either by stabilizing ordered lipid phases or by reducing the line tension between coexisting lipid phases. Higher resolution techniques that probe length scales smaller than the optical wavelength ${ }^{23,32}$ will be essential for confirming this proposal. 


\section{Methods Summary:}

Chemicals. 1,2-dioleoyl-sn-glycero-3-phosphocholine (DOPC), 1,2dipalmitoyl-sn-glycero-3-phosphocholine (DPPC), sphingomyelin(egg) (ESM), cholesterol (ovine wool), GM1 Ganglioside (Brain, OvineAmmonium Salt), and 1,2-dioleoyl-sn-glycero-3-phosphoethanolamine-N(lissamine rhodamine B sulfonyl) (ammonium salt) (Rh-DPPE) were purchased from Avanti Polar Lipids. Cholera Toxin Subunit B (CTXB) conjugated with Alexa647 (A647-CTXB) was purchased from Invitrogen. FITC-Glucomannan was purchased from Carbomer Inc. Hyaluronic Acid (from rooster comb) was purchased from Sigma Aldrich.

Lipid compositions: Fig. 2,4: ESM:DOPC:Cholesterol:Rh-DPPE:GM1 54.8:25:20:0.1:0.1 mol \%, Fig. 3 DPPC:DOPC:Ergosterol:Rh-DPPE:GM1 54.8:25:20:0.1:0.1 mol \%.

Preparation of glycan networks and lipid bilayer patches. $2 \mu \mathrm{l}$ of dilute $(100 \mu \mathrm{g} / \mathrm{ml})$ solutions of glycan dispersed in ultrapure water were deposited on disc-shaped (typically $7.5 \mathrm{~mm}$ in diameter) plasma-oxidized poly(dimethyl)siloxane (ox-PDMS) and allowed to dry on a hotplate set at 65 ${ }^{\circ} \mathrm{C}$. Once the liquid has dried completely, an aqueous buffer (typically $10 \mathrm{mM}$ Tris, $150 \mathrm{mM} \mathrm{NaCl}, 2 \mathrm{mM} \mathrm{CaCl}_{2}, \mathrm{pH}$ 7.5) was added to submerge the surface and rehydrate the glycans. Giant unilamellar vesicles (GUVs) were prepared using the electroformation technique similar to ${ }^{27}$. After a 10 minute incubation interval, $10 \mu \mathrm{l}$ of GUV containing solution (pre-warmed to $65^{\circ} \mathrm{C}$ ) was added to the chamber above the PDMS disk. GUVs sediment onto the glycan network, where they rupture to form 2D supported lipid bilayer patches. After 10 minutes, the hotplate was turned off and the system was allowed to cool to room temperature. Skim milk solution (prepared as a stock solution of $10 \mathrm{mg} / \mathrm{ml}$ in ultrapure water) was added to obtain a final protein concentration of $\sim 0.1 \mathrm{mg} / \mathrm{ml}$ in the chamber and allowed to incubate for at least 10 minutes to block the regions of the surface not covered by lipid bilayer patches. Then $5 \mu \mathrm{l}$ A647-CTXB (prepared as a stock solution of 0.1 $\mathrm{mg} / \mathrm{ml}$ in phosphate buffered saline $\mathrm{pH} 7.5$ ) was added to the chamber to give a final concentration of $\sim 0.05 \mathrm{mg} / \mathrm{ml}$. A647-CTXB labels ordered lipid phases.

Imaging: Supported lipid bilayers were imaged with an upright confocal microscope (Zeiss LSM 510) with a 63x/ 1.0 N.A. water dipping objective. FITC was excited with a $488 \mathrm{~nm}$ laser with the acquisition acousto-optic tuneable filter (AOTF) set at 20 percent. A band pass filter $500-510 \mathrm{~nm}$ was used in the detection channel. Rhodamine-B was excited with a $543 \mathrm{~nm}$ laser with AOTF set at 10 percent. A bandpass filter $565-615 \mathrm{~nm}$ was used in the 
detection channel. CTXB-A647 was excited with the $633 \mathrm{~nm}$ laser, AOTF 30 percent. A band pass filter $650-710 \mathrm{~nm}$ was used in the detection channel. The images were taken sequentially to further reduce cross-talk between the channels. Images were captured at 14-bits.

\section{References:}

1 Alberts, B. et al. Molecular Biology of the Cell. 4 edn, (Garland Science, 2002).

2 Laurent, T. \& Fraser, J. Hyaluronan. The FASEB Journal 6, 23972404, (1992).

3 Masako, O. The ultrastructure of yeast: Cell wall structure and formation. Micron 29, 207-233, (1998).

4 Somerville, C. et al. Toward a systems approach to understanding plant-cell walls. Science 306, 2206-2211, (2004).

5 Marth, J. D. A unified vision of the building blocks of life. Nat. Cell Biol. 10, 1015-1016, (2008).

6 Lingwood, D. \& Simons, K. Lipid rafts as a membrane-organizing principle. Science 327, 46-50, (2010).

7 Brown, D. A. \& London, E. Functions of lipid rafts in biological membranes. Annu. Rev. Cell Dev. Biol. 14, 111-136, (1998).

8 Engelman, D. M. Membranes are more mosaic than fluid. Nature 438, 578-580, (2005).

9 Munro, S. Lipid rafts: Elusive or illusive? Cell 115, 377-388, (2003).

10 Yethiraj, A. \& Weisshaar, J. C. Why are lipid rafts not observed in vivo? Biophysical Journal 93, 3113-3119, (2007).

11 Veatch, S. L. \& Keller, S. L. Organization in lipid membranes containing cholesterol. Phys. Rev. Lett. 89, 268101, (2002).

12 Baumgart, T., Hess, S. T. \& Webb, W. W. Imaging coexisting fluid domains in biomembrane models coupling curvature and line tension. Nature 425, 821-824, (2003).

13 McConnell, H. M. \& Vrljic, M. Liquid-liquid immiscibility in membranes. Annual Review of Biophysics and Biomolecular Structure 32, 469-492, (2003). 
14 Stottrup, B. L., Veatch, S. L. \& Keller, S. L. Nonequilibrium behavior in supported lipid membranes containing cholesterol. Biophysical Journal 86, 2942-2950, (2004).

15 Parthasarathy, R., Yu, C. H. \& Groves, J. T. Curvature-modulated phase separation in lipid bilayer membranes. Langmuir 22, 5095-5099, (2006).

16 Groves, J. T., Ulman, N. \& Boxer, S. G. Micropatterning fluid lipid bilayers on solid supports. Science 275, 651-653, (1997).

17 Tanaka, M. \& Sackmann, E. Polymer-supported membranes as models of the cell surface. Nature 437, 656-663, (2005).

18 Smith, H. L. et al. Model Lipid Membranes on a Tunable Polymer Cushion. Phys. Rev. Lett. 102, (2009).

19 Wong, J. Y. et al. Polymer-cushioned bilayers. I. A structural study of various preparation methods using neutron reflectometry. Biophysical Journal 77, 1445-1457, (1999).

20 Sackmann, E. \& Tanaka, M. Supported membranes on soft polymer cushions: fabrication, characterization and applications. Trends Biotechnol. 18, 58-64, (2000).

21 Watkins, E. B. et al. Structure and Thermodynamics of Lipid Bilayers on Polyethylene Glycol Cushions: Fact and Fiction of PEG Cushioned Membranes. Langmuir 27, 13618-13628, (2011).

22 Wagner, M. L. \& Tamm, L. K. Tethered polymer-supported planar lipid bilayers for reconstitution of integral membrane proteins: Silanepolyethyleneglycol-lipid as a cushion and covalent linker. Biophysical Journal 79, 1400-1414, (2000).

23 Kraft, M. L., Weber, P. K., Longo, M. L., Hutcheon, I. D. \& Boxer, S. G. Phase separation of lipid membranes analyzed with high-resolution secondary ion mass spectrometry. Science 313, 1948-1951, (2006).

24 Yoon, T.-Y. et al. Topographic control of lipid-raft reconstitution in model membranes. Nature Materials 5, 281-285, (2006).

25 Kahya, N., Scherfeld, D., Bacia, K., Poolman, B. \& Schwille, P. Probing lipid mobility of raft-exhibiting model membranes by fluorescence correlation spectroscopy. Journal of Biological Chemistry 278, 28109-28115, (2003). 
26 Kaizuka, Y. \& Groves, J. T. Structure and dynamics of supported intermembrane junctions. Biophysical Journal 86, 905-912, (2004).

27 Subramaniam, A. B., Lecuyer, S., Ramamurthi, K. S., Losick, R. \& Stone, H. A. Particle/Fluid Interface Replication as a Means of Producing Topographically Patterned Polydimethylsiloxane Surfaces for Deposition of Lipid Bilayers. Advanced Materials 22, 2142-+, (2010).

28 Dietrich, C. et al. Lipid rafts reconstituted in model membranes. Biophysical Journal 80, 1417-1428, (2001).

29 Pike, L. J. Rafts defined: a report on the Keystone symposium on lipid rafts and cell function. J Lipid Res 47, 1597-1598, (2006).

$30 \quad$ Young, M. E. et al. The Sur7p Family Defines Novel Cortical Domains in Saccharomyces cerevisiae, Affects Sphingolipid Metabolism, and Is Involved in Sporulation. Molecular and Cellular Biology 22, 927-934, (2002).

31 Malinsky, J., Opekarová, M. \& Tanner, W. The lateral compartmentation of the yeast plasma membrane. Yeast 27, 473-478, (2010).

32 Smith, A. M., Vinchurkar, M., Gronbech-Jensen, N. \& Parikh, A. N. Order at the Edge of the Bilayer: Membrane Remodeling at the Edge of a Planar Supported Bilayer Is Accompanied by a Localized Phase Change. Journal of the American Chemical Society 132, 9320-9327, (2010).

Acknowledgements: We thank the Harvard MRSEC (DMR-0820484), Harvard Center for Brain Science Imaging Facility, the Harvard Center for Nanoscale Systems, and Princeton University for partial support of this research. We also thank S. Lecuyer and M. Staykova for comments on the manuscript.

Author contributions: A.B.S made the initial observation of the effects of glycans on supported lipid membranes. H.A.S and A.B.S. initiated research. A.B.S designed and performed experiments and analysed data. A.B.S, G.G, V.N.M and H.A.S interpreted data and wrote the paper. 


\section{Supplementary Information}

\section{Materials and Methods}

\section{Lipids:}

1,2-dioleoyl-sn-glycero-3-phosphocholine (DOPC), 1,2-dipalmitoyl-snglycero-3-phosphocholine (DPPC), 1-palmitoyl-2-oleoyl-sn-glycero-3phosphocholine (POPC), sphingomyelin(egg) (ESM), sphingomyelin(brain)(BSM), and cholesterol (ovine wool) were purchased from Avanti Polar Lipids. Ergosterol was purchased from Sigma Aldrich.

\section{Lipid membrane fluorescent probes:}

23-(dipyrrometheneboron difluoride)-24-norcholesterol (BODIPY-Chol), GM1 Ganglioside (Brain, Ovine-Ammonium Salt), and 1,2-dioleoyl-snglycero-3-phosphoethanolamine-N-(lissamine rhodamine B sulfonyl) (ammonium salt) (Rh-DPPE) were purchased from Avanti Polar Lipids. Cholera Toxin Subunit B (CTXB) conjugated with Alexa488 and Alexa647 (A488-CTXB, A647-CTXB) and DiI C18:0 were purchased from Invitrogen.

\section{Fluorescently labeled glycans:}

Fluorescein isothiocyanate (FITC)-Dextran 150,000 MW and FITCHyaluronic Acid were purchased from Sigma Aldrich. FITC-Heparan sulfate, FITC-Hyaluronic Acid, FITC-Carboxymethylcellulose and FITCGlucomannan were purchased from Carbomer Inc. FITC-Ficoll was a kind gift from Prof. Krystyn van Vliet (MIT).

Unlabeled glycans:

Hyaluronic Acid (from rooster comb), heparan sulfate, mannan (from yeast), carboxymethylcellulose, Ficoll 400, Ficoll 75, Dextran 150, 000 MW, inulin, pectin, and hydroxypropylcellulose were purchased from Sigma Aldrich.

\subsection{Preparation of giant unilamellar vesicles (GUV)}

Giant vesicles were formed using the electroformation technique ${ }^{1} .6 \mu$ of the desired lipid mixture at a concentration of $1 \mathrm{mg} / \mathrm{ml}$ was spread into a thin film onto a clean indium tin oxide (ITO) coated glass slide using a glass syringe (Hamilton). The slide was placed in a vacuum desiccator for 30 minutes to remove all traces of chloroform. A PDMS gasket was used to construct a barrier around the lipid film, and the slide was placed onto a $65^{\circ} \mathrm{C}$ hotplate (to ensure the lipids were above the miscibility transition temperature). About $600 \mu \mathrm{l}$ of $500 \mathrm{mM}$ sucrose solution was used to hydrate the lipid film, and a second ITO slide was used to close the chamber. The slides were connected to a function generator (Agilent) with conductive copper tape. A sinusoidal AC 
field was applied at a field strength of $0.5 \mathrm{~V} / \mathrm{mm}$ and frequency of $10 \mathrm{~Hz}$ for 1 hour. The giant vesicles were carefully removed from the chamber and suspended in $500 \mathrm{mM}$ glucose solution. GUVs were typically used immediately, and never used more than two days after formation. The lipid compositions that were used for our experiments and the phase state of the membrane are shown in Table 1. Typical images of vesicles are shown in Fig. $\mathrm{S} 2$.

Supplementary Table 1 | Lipid compositions (mole percent) used to prepare lipid membranes.

\begin{tabular}{|c|c|}
\hline Lipid compositions & Membrane phase \\
\hline $\begin{array}{l}\text { ESM:DOPC:Cholesterol:Rh-DPPE:GM1 } \\
\text { 54.8:25:20:0.1:0.1 }\end{array}$ & $\begin{array}{l}\text { Multiphase } \\
\mathrm{L}_{\mathrm{o}}-\mathrm{L}_{\mathrm{d}}\end{array}$ \\
\hline $\begin{array}{l}\text { ESM:DOPC:Cholesterol:Rh-DPPE:GM1 } \\
\text { 54.8:40:5:0.1:0.1 }\end{array}$ & $\begin{array}{l}\text { Multiphase } \\
\mathrm{S}_{\mathrm{o}}-\mathrm{L}_{\mathrm{d}} \\
\end{array}$ \\
\hline $\begin{array}{l}\text { BSM:DOPC:Cholesterol:Rh-DPPE:GM1 } \\
\text { 54.8:40:5:0.1:0.1 }\end{array}$ & $\begin{array}{l}\text { Multiphase } \\
\mathrm{S}_{\mathrm{o}^{-}} \mathrm{L}_{\mathrm{d}}\end{array}$ \\
\hline $\begin{array}{l}\text { ESM:DOPC:Cholesterol:BODIPY-Chol } \\
\text { 54.5:25:20:0.5 }\end{array}$ & $\begin{array}{l}\text { Multiphase } \\
\mathrm{L}_{0}-\mathrm{L}_{\mathrm{d}}\end{array}$ \\
\hline $\begin{array}{l}\text { ESM:DOPC:Cholesterol:BODIPY-Chol } \\
\text { 54.5:40:5:0.5 }\end{array}$ & $\begin{array}{l}\text { Multiphase } \\
\mathrm{S}_{\mathrm{o}^{-}} \mathrm{L}_{\mathrm{d}} \\
\end{array}$ \\
\hline $\begin{array}{l}\text { ESM:POPC:Cholesterol:Rh-DPPE:GM1 } \\
\text { 33:32.8:33:0.1:0.1 }\end{array}$ & $\begin{array}{l}\text { Multiphase } \\
\mathrm{L}_{\mathrm{o}}-\mathrm{L}_{\mathrm{d}}\end{array}$ \\
\hline $\begin{array}{l}\text { ESM:DOPC:Ergosterol:Rh-DPPE:GM1 } \\
\text { 54.8:25:20:0.1:0.1 }\end{array}$ & $\begin{array}{l}\text { Multiphase } \\
\mathrm{L}_{\mathrm{o}}-\mathrm{L}_{\mathrm{d}}\end{array}$ \\
\hline $\begin{array}{l}\text { DPPC:DOPC:Ergosterol:Rh-DPPE:GM1 } \\
\text { 54.8:25:20:0.1:0.1 }\end{array}$ & $\begin{array}{l}\text { Multiphase } \\
\mathrm{L}_{\mathrm{o}}-\mathrm{L}_{\mathrm{d}}\end{array}$ \\
\hline $\begin{array}{l}\text { DOPC:Rh-DPPE:GM1 } \\
\text { 99.8:0.1:0.1 }\end{array}$ & $\begin{array}{l}\text { Single phase } \\
\mathrm{L}_{\mathrm{d}}\end{array}$ \\
\hline $\begin{array}{l}\text { ESM:DOPC:Rh-DPPE:GM1 } \\
\text { 99.8:0.1:0.1 }\end{array}$ & $\begin{array}{l}\text { Single phase } \\
\mathrm{S}_{\mathrm{o}}\end{array}$ \\
\hline $\begin{array}{l}\text { ESM:Cholesterol:Rh-DPPE:GM1 } \\
\text { 69.8:30:0.1:0.1 }\end{array}$ & $\begin{array}{l}\text { Single phase } \\
\mathrm{L}_{0}\end{array}$ \\
\hline
\end{tabular}

\subsection{Preparation of glycan networks}

Since glycan solutions are complex fluids, we hypothesized that instability driven pattern formation, which is known to occur during the drying of other types of complex fluids ${ }^{2,3}$, might be employed to produce such networks. To test our hypothesis, we deposit dilute (typically $100 \mu \mathrm{g} / \mathrm{ml}$ ) solutions of fluorescently labeled glycans dispersed in ultrapure water on disc-shaped (typically $7.5 \mathrm{~mm}$ in diameter) hydrophilic surfaces such as glass slides, 
plasma-oxidized poly(dimethyl)siloxane (ox-PDMS, plasma oxidation produces a layer of hydrophilic silica-like material on the surface of PDMS), or freshly cleaved mica which are all suitable surfaces to support lipid bilayers ${ }^{4-6}$ and allow the liquid to dry. Once the liquid has dried completely, we submerge the surface in an aqueous buffer (typically $10 \mathrm{mM}$ Tris, $150 \mathrm{mM}$ $\mathrm{NaCl}, 2 \mathrm{mM} \mathrm{CaCl}_{2}, \mathrm{pH} 7.5$ ) to rehydrate the glycans. Once prepared, the surface is kept immersed in an aqueous environment at all times (Supplementary Fig. 3 shows a graphical cartoon). Typically, we use PDMS oxidized in air plasma (10 seconds, Harrick Plasma Cleaner/Sterilizer PDC-32G at maximum RF power) as our substrate.

We find that spatially patterned radial networks - homogeneous glycan density in the center transitioning to inhomogeneous regions with of high and low glycan density towards the edges - are produced. The shape and interdomain spacing in the inhomogeneous region varies with position on the disk and the drying conditions. The structure of the glycan network remains unperturbed upon subsequent gentle addition and quiescent submersion in aqueous buffers. Note however that we observe fluorescence signal in the buffer which suggests that some glycans do come off the surface upon addition of buffer. These free glycans in solution likely reabsorb onto any bare hydrophilic regions (due to the high surface energy of such surfaces ${ }^{7}$ ), and thus all surfaces have a coating of glycan. Using this method, hydrated glycan networks of radially modulated lateral density can be reproducibly obtained from a wide variety of biologically-derived, fluorescently-derivatized and synthetic glycans.

The submerged layer is stable for at least 48 hours, and the hydrated glycans remain anchored to the surface and do not diffuse under quiescent conditions. This observation is important since the absence of diffusion allows us to consider any subregion of the glycan layer as a connected yet independent glycan network. Thus, using this method, we readily obtain $O(1000) 100 \mu \mathrm{m}^{2}$ independent surface-supported hydrated glycan networks on a single disk.

\subsection{GUV fusion and cholera toxin labeling}

After a 10 minute incubation interval, $10 \mu \mathrm{l}$ of a $500 \mathrm{mM}$ glucose/sucrose solution containing giant vesicles (pre-warmed to $65^{\circ} \mathrm{C}$ ) is added to the chamber above the PDMS disk. The high density of the sugar solution ensures rapid sedimentation of the vesicles onto the glycan surface. The chamber is covered with an $18 \mathrm{~mm}$ diameter circular coverslip, to prevent excessive evaporation of the buffer, and the sample is left to incubate for 10 minutes on the hotplate. Giant vesicles sediment onto the glycan network, where most vesicles rupture and form $2 \mathrm{D}$ supported lipid bilayer patches. After 10 minutes, the hotplate is turned off and the slide is allowed to cool to room 
temperature. The coverslip is removed and the surface is gently washed by pipetting fresh Tris- $\mathrm{HCl}$ buffer over the surface to remove any unfused vesicles.

When Cholera Toxin subunit B (CTXB) is used to label the bilayer, the surface is blocked post vesicle fusion to reduce non-specific adsorption of the protein onto regions devoid of lipids. Skim milk solution (prepared as a stock solution of $10 \mathrm{mg} / \mathrm{ml}$ in ultrapure water) is added to obtain a final protein concentration of $\sim 0.1 \mathrm{mg} / \mathrm{ml}$ in the chamber and allowed to incubate for at least 10 minutes. The skim milk block is inspired by the blocking procedure used for Western blots. We find that skim milk is a superior block compared to the more common bovine serum albumin block (background fluorescence is extremely low).

$5 \mu \mathrm{l}$ of CTXB (prepared as a stock solution of $0.1 \mathrm{mg} / \mathrm{ml}$ in phosphate buffered saline $\mathrm{pH} 7.5$ ) is added to the chamber to give a concentration of $0.05 \mathrm{mg} / \mathrm{ml}$. A fresh $18 \mathrm{~mm}$ coverslip is used to cover the chamber and the preparation is allowed to incubate for at least 30 minutes before visualization with the confocal microscope.

For samples without CTXB the skim milk block step was omitted. The presence or absence of skim milk did not affect the behavior of the lipid bilayers or the glycan network.

\subsection{Surface imaging}

Supported lipid bilayers were imaged with an upright confocal microscope (Zeiss LSM 510) with a 63x/ 1.0 N.A. water dipping objective. FITC was excited with a $488 \mathrm{~nm}$ laser with the acquisition acousto-optic tunable filter (AOTF) set at 20 percent. A band pass filter 500-510 nm was used in the detection channel. Rhodamine-B was excited with a $543 \mathrm{~nm}$ laser with AOTF set at 10 percent. A bandpass filter $565-615 \mathrm{~nm}$ was used in the detection channel. CTXB-A647 was excited with the $633 \mathrm{~nm}$ laser, AOTF 30 percent. A band pass filter $650-710 \mathrm{~nm}$ was used in the detection channel. The images were taken sequentially to further reduce cross-talk between the channels. Images were captured at 14-bits.

\subsection{Image analysis}

The function 'tiffread29' was used to open the native '.1sm' files in the Matlab environment ${ }^{8}$. Each '.lsm' file consisted of the glycan image, $\mathrm{L}_{\mathrm{d}}$ image and $\mathrm{L}_{\mathrm{o}}$ image. For each file, the $\mathrm{L}_{o}$ and $\mathrm{L}_{d}$ images were summed. Pixel intensities that were less than the mean intensity of this summed image was then set to zero in all three images which clears regions that are not covered by the lipid bilayer. Then the images were automatically reduced to obtain a square region 
of interest (ROI) that inscribes the bilayer. These images were used for all of our subsequent analysis. All analysis was performed with custom programs written in Matlab.

\subsection{Power spectrum density (PSD) analysis}

A 2D Hamming window was applied to the ROI to reduce edge effects and a Fast Fourier Transform (FFT) of the image is calculated. A radial average of this FFT was then calculated to obtain the power spectral density. A smoothing function (moving average method with a span of 3) was applied to reduce noise in the PSD curves, and the PSDs of the different channels were normalized by dividing with the mean intensity value of the respective channels.

\subsection{Cross-correlation analysis}

The mean intensity value of the channels was subtracted from each pixel and a 2D Hamming window was applied. The built-in Matlab function normxcorr 2 was used to calculate the normalized cross correlation matrix between the glycan channels and $\mathrm{L}_{\mathrm{o}}$ channels, and, the glycan channels and the $\mathrm{L}_{\mathrm{d}}$ channels. We calculate the half-radial average of the cross-correlation matrix corresponding to the positive and negative half-space of the lags and patch the two curves about zero to obtain the 1D plots of the cross-correlation versus lag.

\subsection{Intensity contour plots}

Each pixel was normalized by subtracting the mean and dividing by the standard deviation of the ROI intensity. Glycan channel and $\mathrm{L}_{0}$ channel, glycan channel and $\mathrm{L}_{\mathrm{d}}$ channel pixel pair intensities were aggregated and the Matlab routine contourf was called to plot contour plots of the fluorescence intensities.

\section{Note about FRET between FITC and rhodamine}

The emission spectrum of FITC and excitation spectrum of rhodamine sufficiently overlap that Forster resonance energy transfer (FRET) is possible when the two fluorophores are within the Forster radius ${ }^{9}$. Our confocal images and experimental setup of bilayer patches are designed to allow for FRET to be detected. Indeed, significant quenching of the FITC (FRET donor) bound to the bilayer is evident when a membrane containing Rh-DPPE (FRET acceptor) is adsorbed onto a uniform glycan network (Fig. 4 of the main paper). This reveals that the bilayer is in close contact with the glycan network. 
Note that FRET occurs to a lesser extent on raft-forming membranes on inhomogeneous networks since the rhodamine-molecules partition to glycan poor regions. Dark regions in the glycan network are not due to quenching since glycan patterns are continuous from regions that contain the acceptor and regions that do not contain the acceptor (See Fig. 2) of the main paper.

\subsection{Fluorescence recovery after photobleaching (FRAP) measurement of the diffusion coefficient of phases}

Diffusion of fluorescent probes in a bilayer is another independent means of determining phase identities. In general, diffusion of fluorescent probes is about 1-10 times slower in the $\mathrm{L}_{\mathrm{o}}$ phase compared to the $\mathrm{L}_{\mathrm{d}}$ phase due to the increased viscosity of the ordered phase ${ }^{10}$. Lipids are immobile in the $S_{o}$ phase ${ }^{10}$.

We use the fluorescent lipid probe BODIPY-Chol which partitions equally into $L_{o}, L_{d}$ and $S_{o}$ phases ${ }^{11}$ and so allows FRAP measurements of diffusion in all phases with the same molecule.

\subsection{Identifying lipid domains in multiphase membranes labeled with an equipartitioning probe}

Since BODIPY-Chol partitions evenly between phases (Supplementary Fig. $4 a$ ), determining the location of domains in order to conduct FRAP measurements required some creativity.

We recognize the fact that probe molecules are constantly being photobleached when exposed to the laser, and that the rate of bleaching is dependent on the amount of laser light to which each probe molecule is exposed. In a confocal microscope, the laser rasters over an array of pixels with a characteristic dwell time (typically $3.2 \mu$ s for the FRAP experiments presented here). For the extreme case of an immobile fluorophore, each fluorophore in a pixel will be exposed to a laser light pulse for $3.2 \mu$ s each time a frame is acquired. Mobile fluorophores however can diffuse out of the laser path and thus, on average, are exposed to a shorter pulse of laser light. Since probe molecules diffuse more slowly in viscous domains, fluorophores in membranes with domains of differing viscosities should exhibit regions of differing bleach rates.

We test the above idea with multiphase membranes on inhomogeneous glycan networks and find that when images are acquired with full laser intensity (to encourage photobleaching) on an initially uniformly fluorescent membrane, domains are revealed after several passes of the laser (Supplementary Fig. 4 ad). An intensity profile of a strip on the image reveals that the membrane as a whole is bleached with each pass of the laser (i.e. the total fluorescence 
intensity decreases), but domains in the membrane exhibit different rates of photobleaching (Supplementary Fig. 4e). Similar experiments on homogeneous glycan networks do not reveal any domains. These experiments provide an indication of the existence of compartmentalized domains of different viscosities in membranes adjacent to inhomogeneous glycan networks.

\subsection{Quantitative determination of diffusion coefficients}

We saved the coordinates of the identified domains in the confocal software, and we allow membrane fluorescence to recover completely. Note that the bleached bilayer is a small fraction of the whole bilayer patch and thus by waiting for an interval of 20 minutes, unbleached fluorophores diffuse into our region of interest. All FRAP experiments are performed identically as follows: 63X 1.0 NA water dipping objective, laser line: $488 \mathrm{~nm}$ at 50 percent power, image acquisition AOTF setting 0.5 percent, bleach AOTF setting 100 percent, bleach pulse $105 \mathrm{~ms}$, acquisition frame rate $105 \mathrm{~ms}$. ROI 50 x 50 pixels, pixel size $79.8 \mathrm{~nm}$. A circular bleach spot is defined at the center of the ROI with a nominal radius of 5 pixels. Bleaching was carried out four times per ROI, and we were careful to allow sufficient time for full recovery to occur before initiating the next bleach acquisition (typically by moving to 20 other positions before coming back to the first position to carry out another round of bleaching). All images were normalized by dividing the intensity at each pixel with the average intensity of the first pre-bleach image. Acquisition photobleaching correction was done by dividing the intensity of each image post-bleach with a reference time series where a bleach pulse was not applied.

The four sets of images acquired for each ROI was averaged and the mean intensity in the bleached region versus time was obtained with the regionprops routine in Matlab. The fluorescence recovery data were fitted for a 'characteristic' diffusion time, $\tau$, in the linear least-squares sense with the well-known Axelrod series solution ${ }^{12}$ for a Gaussian intensity profile:

$$
F(t)=A \sum_{n=0}^{\infty}{\frac{(-K)^{n}}{n !}}^{n}(1+n(1+2 t / \tau))^{-1}
$$

where $A$ is a parameter that depends on the bleach geometry and laser power in the static laser setup (not well defined for the confocal setup, and thus left as a free parameter), and $K$ is the bleach depth. Diffusion coefficients, $D$ are calculated using $D=\frac{R^{2}}{4 \tau}$.

FRAP measurements were performed in the $\mathrm{L}_{\mathrm{d}}$ and $\mathrm{L}_{\mathrm{o}}$ domains and the calculated diffusion coefficients are plotted in Supplementary Fig. 5a. Diffusion of BODIPY-Chol is $\sim 3$ times faster in the $\mathrm{L}_{\mathrm{d}}$ phase. The quantitative difference in diffusion of the probe in the membrane, along with 
the qualitative demonstration above, provides a clear indication that two distinct membrane phases exist on multiphase bilayers supported on inhomogeneous glycan networks. FRAP measurements of multiphase lipid bilayers on homogenous glycan networks where macroscopic domains do not form yield a diffusion coefficient that is intermediate between the values obtained for the phase-separated membrane (Supplementary Fig. 5a). The fluorescence intensity of $S_{o}$ domains does not recover, which confirms the absence of lateral diffusion in the gel phase (Supplementary Fig. 5b).

Note that these measurements explicitly confirm that the lipids are fully mobile in the bilayer.

\section{Controls for fluorescence labeling}

Numerous experiments were performed with unlabeled glycans. In all cases, similar to glycans labeled with FITC, multiphase membranes show large time stable domains on inhomogeneous glycan networks and no domains on homogeneous networks.

Rh-DPPE (selectively partitions into $\mathrm{L}_{\mathrm{d}}$ domains), BODIPY-Chol (partitions equally into $L_{d}$ and $L_{o}$ domains), Alexa647-Cholera Toxin B bound to GM1 (partitions into $\mathrm{L}_{\mathrm{o}}$ domains and $\mathrm{S}_{\mathrm{o}}$ domains), and DiI C18:0 (partitions into $\mathrm{L}_{\mathrm{d}}$ domains) were used to label the membranes respectively either singly or in combination. Our results indicate that the choice of fluorescent label does not modify the behavior of lipid membranes on glycan networks. Importantly membranes without the glycolipid GM1 and CTXB also show the same behavior on glycan networks.

\section{Microscale roughness measurements of the glycan networks}

There is a report that microstructral roughness due to the chemical etching of silica surfaces can pattern the formation of domains in membranes showing $\mathrm{L}_{\mathrm{o}}-\mathrm{L}_{\mathrm{d}}$ coexistence. The more rigid $\mathrm{L}_{\mathrm{o}}$ domains coarsen on smooth regions while the less rigid $\mathrm{L}_{d}$ domains coarsen on the rougher regions ${ }^{13}$. While pioneering in its experimental demonstration of microstructural effects, note however that the formation of the domains was extremely slow ( 72 hours or more) and it was unclear if the domains exhibit thermal reversibility ${ }^{13}$. In contrast, membranes on our glycan coated surfaces exhibit thermal reversibiliy and form within minutes of quenching.

Nevertheless, putative differences in roughness might be a starting point for a mechanistic explanation of glycans effects on lipid membranes. We image our glycan networks with an MFP-3DCoax Atomic Force Microscope (AFM) coupled with an inverted microscope (Asylum Research, Santa Barbara, CA). Silicon nitride AFM probes model AC240TS (Olympus, Japan) 
were used. Typical images of the inhomogeneous and homogeneous networks are shown in Fig. S6A,C. Root mean square roughness $\left(\mathrm{R}_{\mathrm{RMS}}\right)$ of the glycanrich domains and the glycan-poor domains were measured with a built-in function in the MFP-3D software. The $\mathrm{R}_{\mathrm{RMS}}$ values were calculated over a $1 \mathrm{x}$ 1 micron square region to allow comparison of the roughness values between inhomogeneous glycan networks (we placed the sampling box over glycanrich domains and glycan-poor domains of sufficient size to fully enclose the 1 $\mathrm{x} 1$ micron box). For homogenous networks where there are no domains to choose from and for bare PDMS, the sampling box was placed at random. We found that the $\mathrm{R}_{\mathrm{RMS}}$ between the glycan-rich and glycan-poor regions were similar within experimental error (Supplementary Fig. 6b). RRMS of the homogeneous glycan networks were also indistinguisable within experimental error (Supplementary Fig. 6d). Thus it is clear that the effects of glycans are distinct from the roughness effects reported by Yoon et al. ${ }^{13}$.

\section{Confirmation that glycan poor domains of inhomogeneous glycan networks are not devoid of glycans}

Evidence that glycan-poor regions of inhomogeneous glycan networks are indeed glycan poor and not devoid of glycans are as follows. (i) Roughness measurements (Supplementary Fig. 6d) indicates that the $\mathrm{R}_{\mathrm{RMS}}$ of glycan poor regions is different from $\mathrm{R}_{\mathrm{RMS}}$ of bare oxidized PDMS. (ii) We use a thresholding routine in Matlab, graythresh, to define automatically the location of glycan rich domains and glycan poor domains. The normalized mean fluorescence intensities of these domains in $n=15$ glycan networks was then calculated and shown in Supplementary Fig. 7a. It is clear that the fluorescence intensity in glycan poor regions is not zero. (iii) AFM phase imaging is sensitive to the presence of different chemical groups on a surface ${ }^{14}$. We obtain a phase image along with the height profile of a section along the boundary of a glycan rich and glycan poor domain of an inhomogeneous glycan network. We show a 3D reconstruction of the topography of this section and overlay the phase information. The phase image was false colored according to the phase angle. In the phase image glycans appear yellow while the substrate appears purple. It is clear that glycans are present at a lower density in the glycan poor regions. Here we confirm at the molecular level that glycans are present in the glycan poor domains of inhomogeneous networks.

\section{Controls for gross topographic effects}

The glycan-rich regions are raised with respect to the glycan-poor regions (Supplementary Fig. 6a). Naively, gross changes in topography might explain our observation of phase patterning in multiphase membranes. How do we untangle putative gross topographical effects from other chemical or microstructural features of the glycan network? We devised a method to test 
for the effects of topography by making replicas of the glycan network in PDMS. These replicas preserve the topography of the network while being composed purely of inert PDMS. We modify our previously reported method of making replicas of particle-covered interfaces ${ }^{6}$ to make a copy of the glycan network. Briefly, UV curable epoxy (UVO-110, Epotek) was poured and then cured on a PDMS disk with a prepared glycan network. The PDMS is peeled off the hardened epoxy, which now has an imprint of the topography of the glycan network. We found that it was important to sonicate the mold 34 times with ethanol and water for 20 minutes each to remove most traces of glycan from the epoxy. The epoxy mold was then used to produce all-PDMS surfaces (Supplementary Fig. 8). We discard the first three castings since we found traces of glycan remaining on the cured PDMS (we found that the FITC signal from the glycan could be detected on these PDMS surfaces despite the extensive sonication) and use the fourth and subsequent surfaces for our experiments.

We deposit multiphase membranes on the PDMS replicas and visualize with the confocal microscope (Supplementary Figure 8). Fourier analysis, similar to the ones performed for the glycan networks, was performed on these images. Note however that we use confocal reflection microscopy to visualize the replica, since it is not fluorescent. Thus gray-scale intensity of the images of the replica corresponds to the height and curvature (topography) of the surface, and not the number density of glycans on the surface (which is what is measured in the fluorescence images). Fourier analysis allows the extraction of characteristic length scales of the all-PDMS replica and any domains in the membrane (Supplementary Fig. 8c,d). It is clear from the images, the PSDs, the cross-correlation analysis, and the intensity contour plots that the replica does not pattern the phase separation of the membrane. It might be possible that there are differences at the nanoscale regarding the contours of the lipid bilayers and the substrates, but it is unlikely to be a main factor given the many papers that report lipid bilayers follow faithfully the topography of even highly rough silica substrates ${ }^{15-17}$. We thus conclude that the gross topography of the glycan network alone cannot pattern domains in multiphase membranes. 


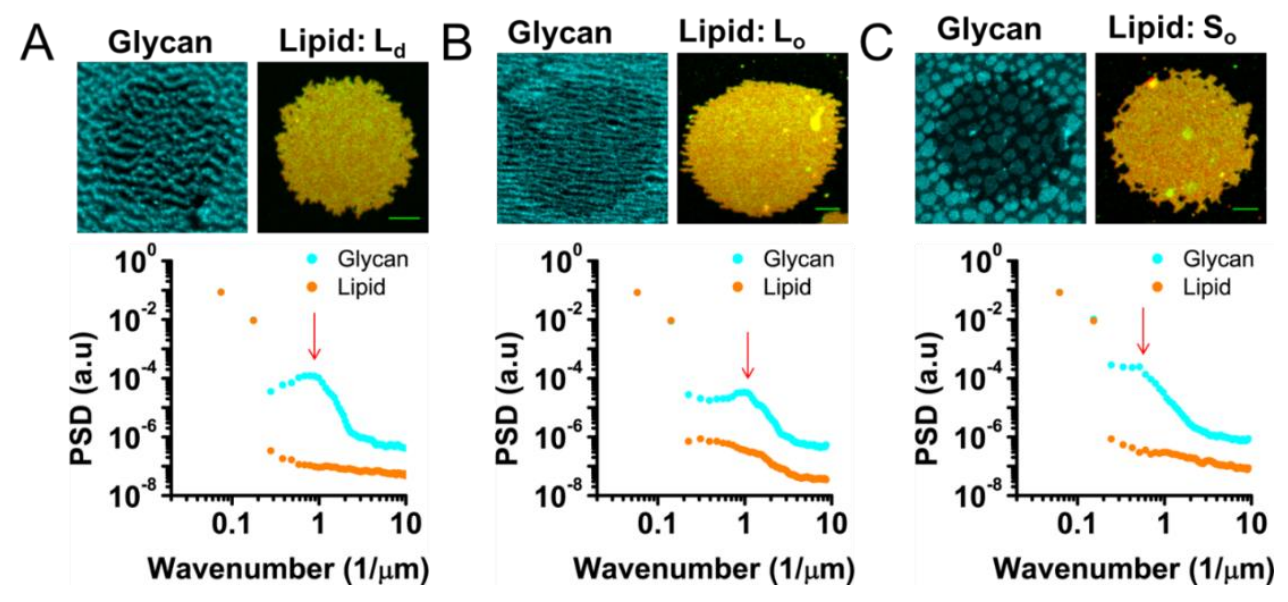

Supplementary Figure 1 | Single-phase membranes are insensitive to glycan networks. Images are multichannel confocal fluorescence micrographs. Lipid images are composites of Rh-DPPE channels, false colored red, and A647-CTXB channels, false colored green. Glycan images are false colored cyan. PSDs of the glycan networks are shown as cyan filled circles while those of the lipid bilayers are shown as orange filled circles. Red arrows indicate peaks of interest denoting the dominant length scale (if present) in the curves. Bilayers were imaged at $\mathrm{T}=23^{\circ} \mathrm{C}$. (a-c) Confocal images indicate the absence of domains in single phase $\mathrm{L}_{\mathrm{d}}, \mathrm{L}_{\mathrm{o}}$ and $\mathrm{S}_{\mathrm{o}}$ resting on inhomogeneous glycan networks. This is confirmed by the lack of a characteristic length scale peak in the lipid PSDs despite the pronounced peak in the glycan PSDs. We conclude that glycans cannot pattern the formation of lipid domains independent of favorable lipid-lipid interactions, and that the lipid phase-sensitive probes that we use do not interact directly with the glycans. Scale bars $5 \mu \mathrm{m}$.
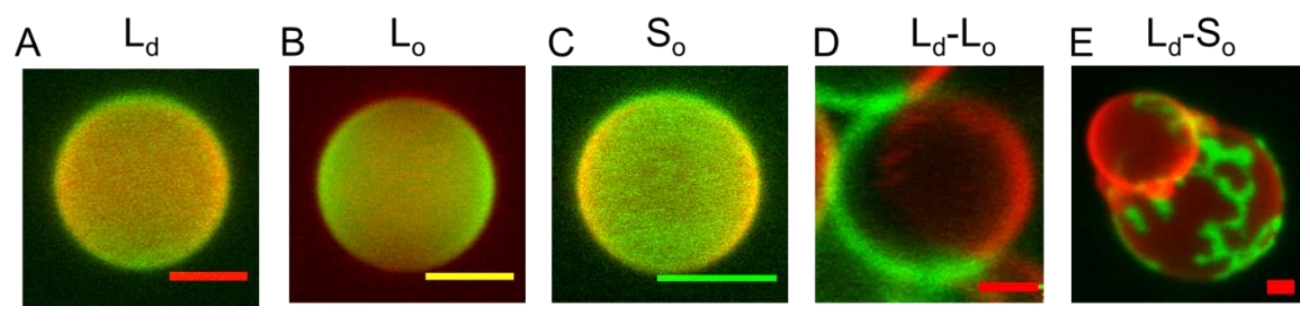

Supplementary Figure $2 \mid$ Representative images of the giant vesicles used to obtain membrane patches on our glycan networks. Vesicles were imaged at $\mathrm{T}=23^{\circ} \mathrm{C}$. The membranes were labeled with Rh-DPPE (false colored red) and A647-CTXB bound to GM1 (false colored green). Images shown here are composites of the red and green channels. (a-c) In single phase membranes the fluorescent probes distributes uniformly in the membranes. (d, e) In multiphase membranes the probes partition into distinct micron scale compartments, which indicates the presences of multiple phases. In vesicles showing $\mathrm{L}_{0}-\mathrm{L}_{\mathrm{d}}$ coexistence the domains appear circular while in vesicles showing $S_{o}-L_{d}$ coexistence domains have irregular shapes. Prior to fusion onto glycan networks the vesicles were heated to $65^{\circ} \mathrm{C}$ which melts the domains in the multiphase membranes. Scale bars $5 \mu \mathrm{m}$. 

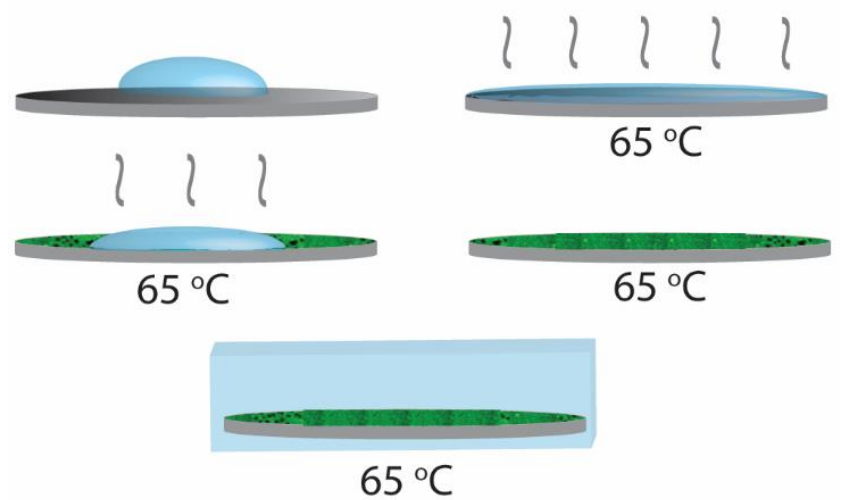

Supplementary Figure 3 | Schematic of the preparation procedure of glycan networks on oxidized PDMS. Glycans were dissolved in ultrapure water to a concentration of $1 \mathrm{mg} / \mathrm{ml}$. Stock solutions were diluted 10 times to produce working solutions at a concentration of 0.1 $\mathrm{mg} / \mathrm{ml}$. Except for dextran which was used at a concentration of $1 \mathrm{mg} / \mathrm{ml}$, all other glycans were used at a concentration of $0.1 \mathrm{mg} / \mathrm{ml} .2 \mu \mathrm{l}$ of the glycan solution was deposited onto the plasma-oxidized PDMS disk, $7.5 \mathrm{~mm}$ in radius. The dilute glycan solution spreads completely over the hydrophilic PDMS surface. The slide is placed on a $65^{\circ} \mathrm{C}$ hotplate for 3 minutes. The liquid dries, depositing a network of glycan onto the PDMS. $1000 \mu \mathrm{l}$ of prewarmed $\left(65^{\circ} \mathrm{C}\right)$ Tris-HCl buffer is added to the chamber to rehydrate the deposited glycan network and also to prepare the surface for vesicle fusion.
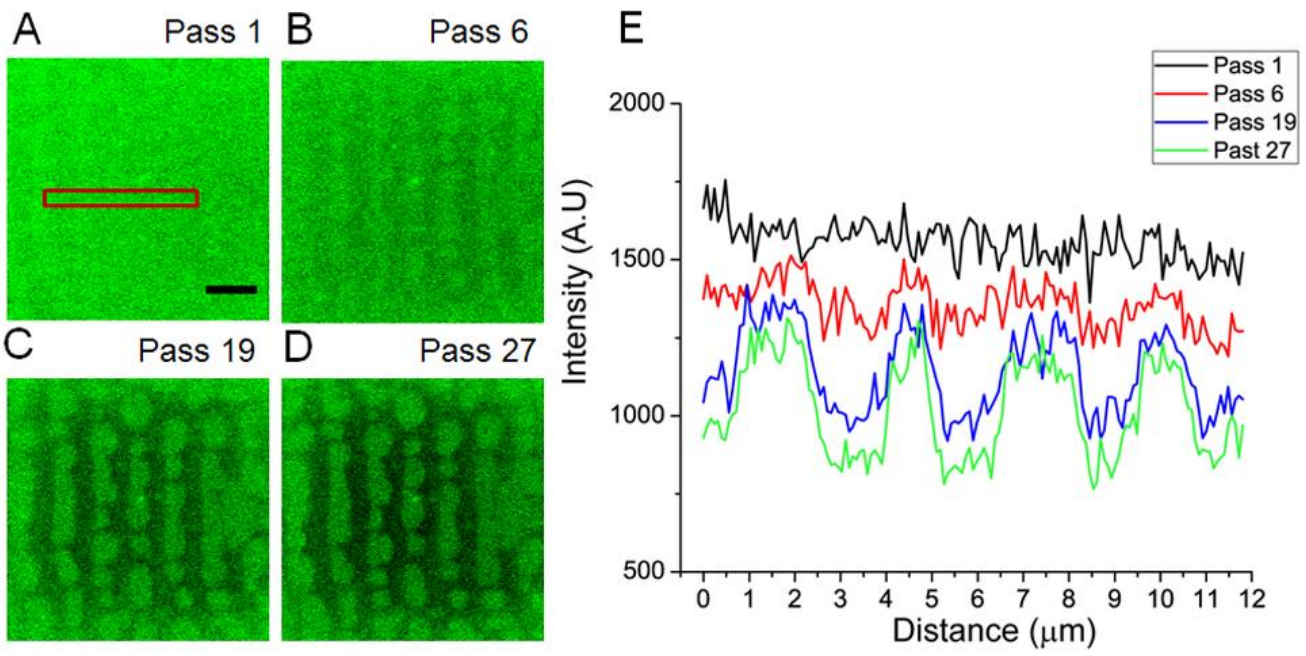

Supplementary Figure 4 | Identifying lipid domains in multiphase membranes labeled with an equipartitioning probe. (a) Typically there is little to no contrast in multiphase membranes on inhomogeneous glycan networks labeled with Bodipy-Chol, since the probe does not partition into a specific phase. (b-d) Since the laser rasters at a constant rate to obtain an image, slowly diffusing probe molecules (i.e. probes in more viscous regions of the membrane) have a higher residence time in the laser beam, causing a larger bleach rate in these regions. By scanning over the region several times, viscous phases become darker. (e) Strip intensity profiles (strip chosen shown in (a)) with each pass of the laser, showing the emergence of contrast in the bilayer. 

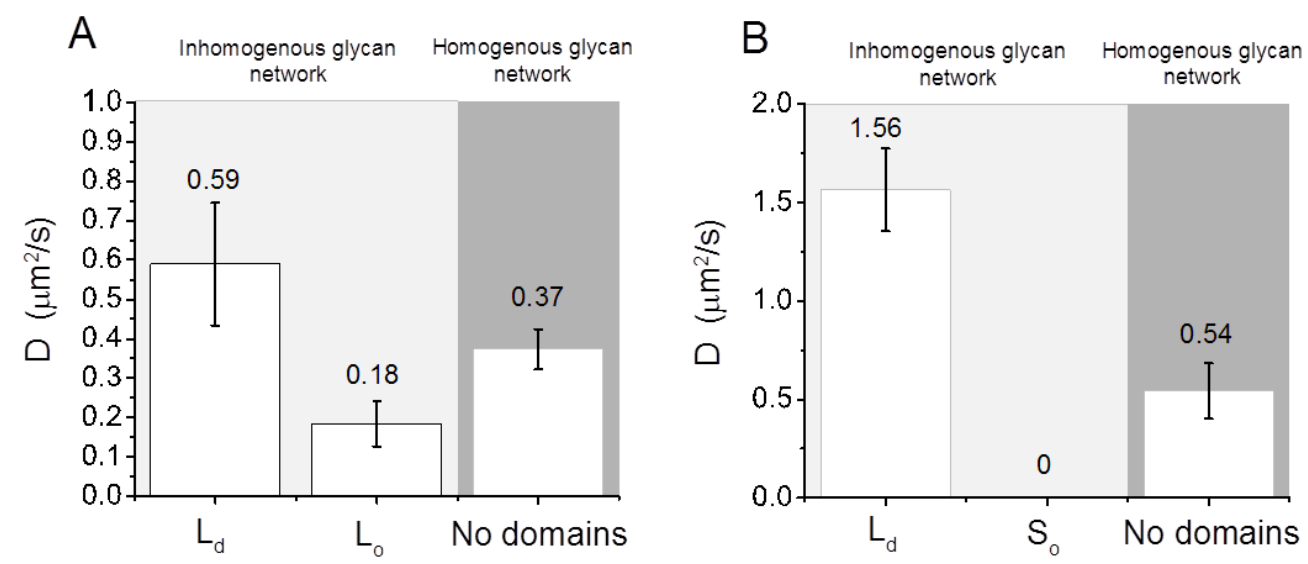

Supplementary Figure 5 | Quantitative determination of lipid mobility of multiphase membranes on glycan networks. (a) Lipids diffuse $\sim 3$ times faster in $L_{d}$ domains on inhomogeneous glycan networks. Membranes with the same composition on homogeneous glycan networks do not show domains. The measured diffusion coefficient in these membranes is intermediate to that of the $\mathrm{L}_{d}$ and $\mathrm{L}_{0}$ phases present on inhomogeneous glycan networks. (b) Lipids are immobile in the solid phase $\left(\mathrm{S}_{\mathrm{o}}\right)$ on inhomogeneous glycan networks, while the lipids are fully mobile in the $\mathrm{L}_{\mathrm{d}}$ phase. Once again the diffusion coefficient of the lipids on homogeneous glycan networks is intermediate to the phases found on inhomogeneous glycan networks. Error bars are standard deviations for $n=20$ measurements.
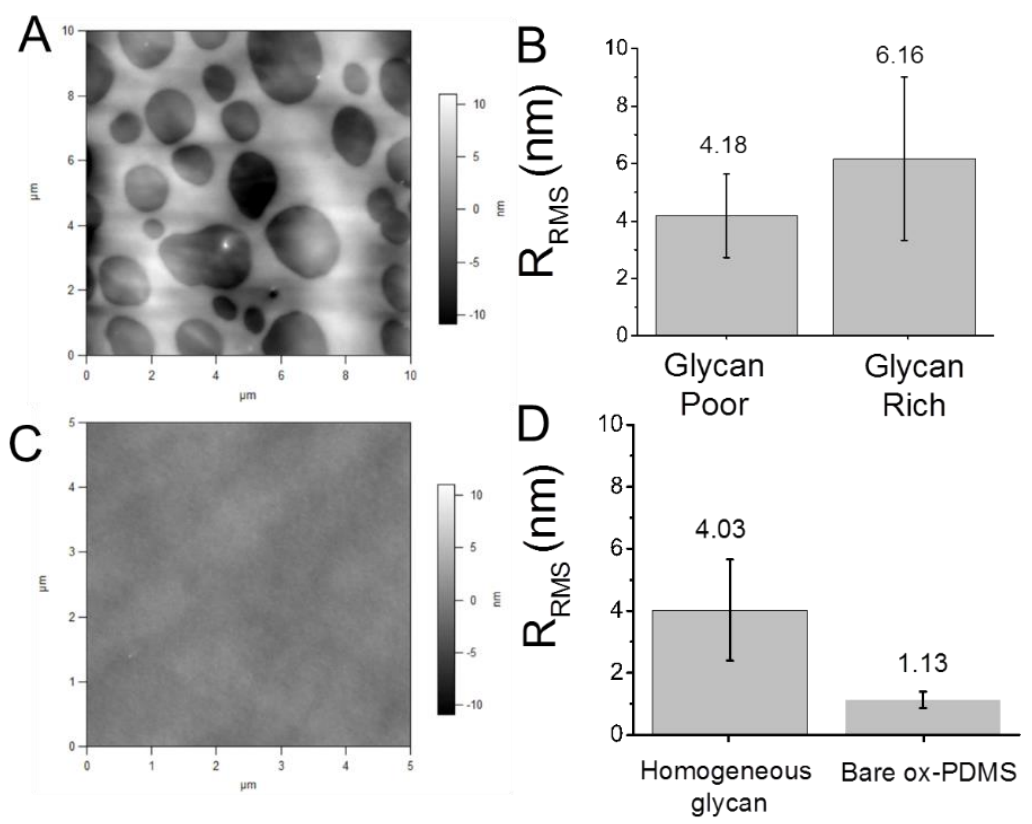

Supplementary Figure 6 | Measurements of root mean square roughness (RRMs) using AFM. (a) AFM image of a typical inhomogeneous glycan network, in this case hyaluronic acid. The dark gray regions are glycan-poor domains and the light gray regions are glycanrich domains. (b) $R_{R M S}$ values indicate no significant difference in roughness between the glycan-rich and glycan-poor regions. (c) AFM image of a typical homogeneous glycan network. (d) $R_{\text {RMS }}$ of homogeneous networks is comparable to those measured on inhomogeneous networks. Bare ox-PDMS (i.e. substrate devoid of glycans) is significantly smoother with a mean $\mathrm{R}_{\mathrm{RMS}}$ value of $1.13 \mathrm{~nm}$. Error bars are standard deviations for $n=10$. 
A

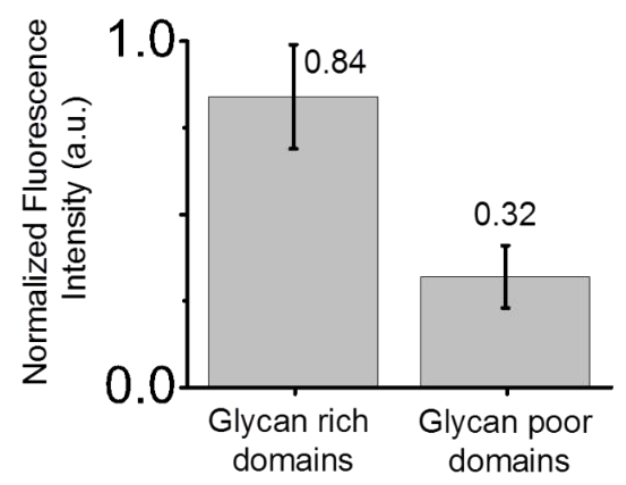

B

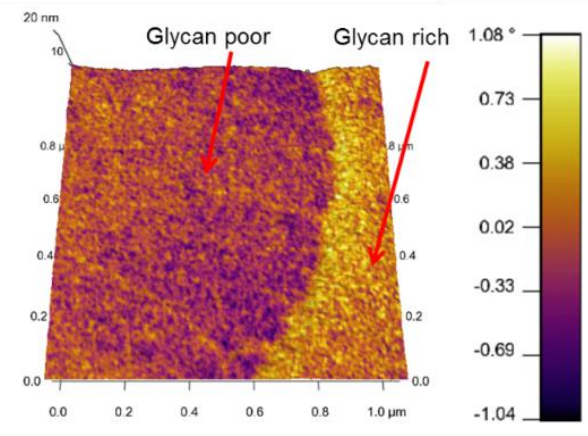

Supplementary Figure 7 | Confirmation that glycan poor domains are not devoid of glycans. (a) If glycans were not present in the glycan poor domains the mean fluorescene intensity should be zero in these regions. We find clearly that the fluorescence intensity in glycan poor regions is not zero thus confirming through optical means that glycans are present in these regions. Furhtermore we find that glycan rich regions have $\sim 2 \mathrm{x}$ the density of glycans per unit area of the surface. Error bars are standard deviations for $n=15$ glycan networks. (b) AFM phase imaging is sensitive to the presence of different chemical groups on a surface ${ }^{14}$. We obtain a phase image along with the height profile of a section of the inhomogeous glycan network. We show a 3D reconstruction of the topography of our glycan network and overlay the phase information. The phase image was false colored according to phase angle. In the phase image glycans appear yellow while the substrate appears purple. It is again clear that glycans are present at a lower density in the glycan poor regions. 

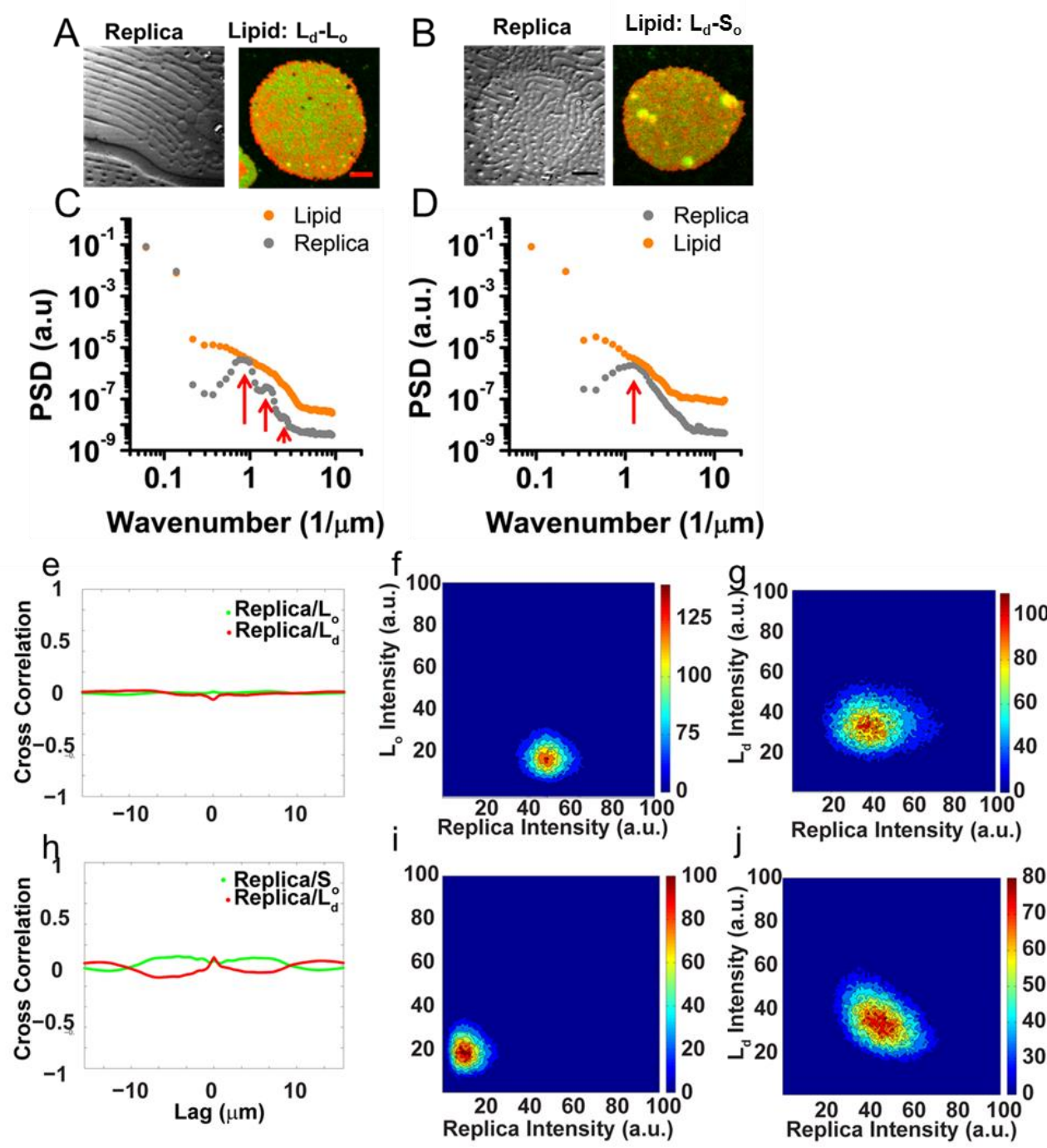

Supplementary Figure $8 \mid$ Topography does not pattern the membrane. We made a replica of the inhomogeneous glycan network in PDMS to produce surfaces that have a topographic profile similar to the networks yet lacking glycan. The replica is composed entirely of PDMS and there are no glycans on the surface. We then deposited bilayers under the same conditions as on glycan networks and prepare similar analysis as shown Figure 2 and 4 of the main paper. The replica surface, which is not fluorescent, was imaged through confocal reflection microscopy (glycans cannot be imaged through reflection, likely because the highly hydrated layers lack optical contrast with the surrounding water). (a,b) Multiphase membranes deposited on the PDMS replicas. Bright yellow circles on the membrane are lipid vesicles adhering to the bilayer patch. (c-d) PSDs of the bilayer (filled orange circles) show that the lipids do not respond to the dominant length scale of the PDMS replica (filled gray circles). (e,h) Radial averages of the normalized cross-correlation matrices of the replica and $\mathrm{L}_{\mathrm{o}} / \mathrm{S}_{\mathrm{o}}$ images (green filled circles) and the replica and $\mathrm{L}_{\mathrm{d}}$ images (red filled circles). No clear correlations are evident. (f,g,i,j) Contour plots of the grey-scale intensity of $\mathrm{L}_{\mathrm{o}} / \mathrm{S}_{\mathrm{o}}$ preferring probes vs. the image of the replica and $L_{d}$ sensitive probes vs. the image of the replica show a cluster of points in intensity phase space. We thus conclude that gross topographic effects cannot explain our observation of membrane patterning. Scale bars $5 \mu \mathrm{m}$. 

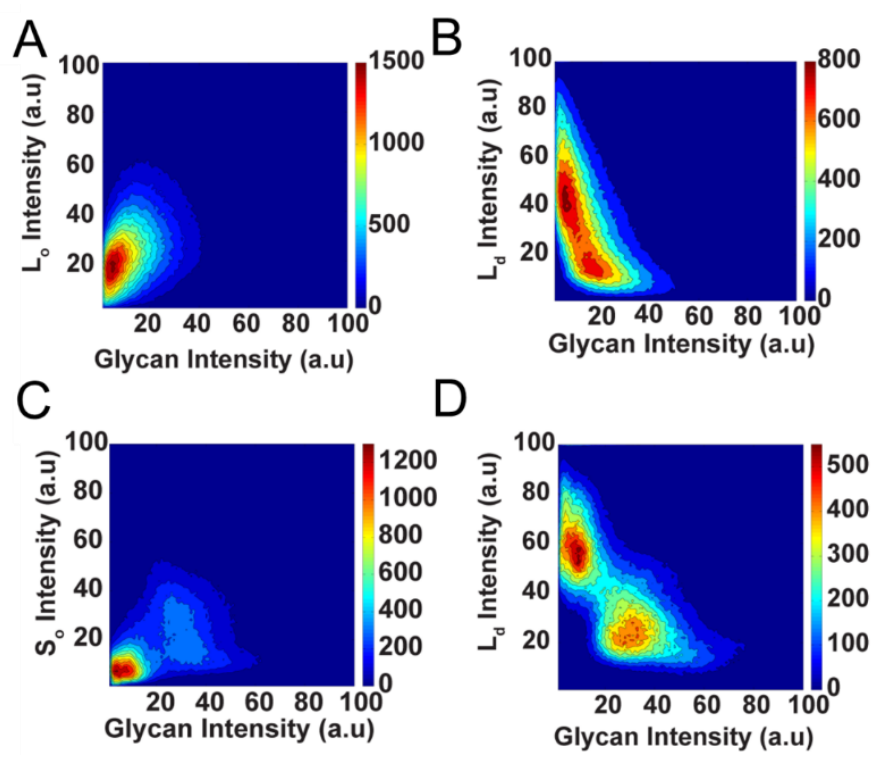

Supplementary Figure 9 | Aggregated intensity contour plots for $(\mathbf{a}, \mathbf{b}) n=15 \mathrm{~L}_{0}-\mathrm{L}_{\mathrm{d}}$ membranes on inhomogeneous glycan networks. (c-d) $n=15 \mathrm{~S}_{0}-\mathrm{L}_{\mathrm{d}}$ membranes on inhomogeneous glycan networks.

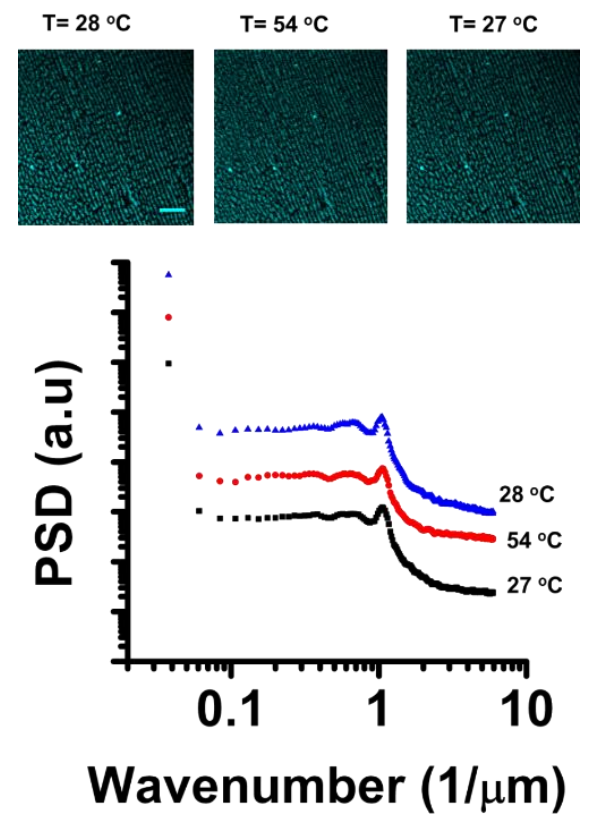

Supplementary Figure 10 | The lateral configuration of the glycan network does not vary significantly with increase in temperature. Fluorescently labeled glycan networks were subject to heating and cooling cycles. We find that the characteristic length scale of the network, as seen here from the peak in the PSD, does not change. 


\section{Supplementary References:}

1 Angelova, M. I. \& Dimitrov, D. S. Liposome Electroformation.

Faraday Discuss. 81, 303-+, (1986).

2 Deegan, R. D. Pattern formation in drying drops. Physical Review E 61, 475, (2000).

3 Rabani, E., Reichman, D. R., Geissler, P. L. \& Brus, L. E. Dryingmediated self-assembly of nanoparticles. Nature 426, 271-274, (2003).

4 Tamm, L. K. \& McConnell, H. M. Supported phospholipid-bilayers. Biophysical Journal 47, 105-113, (1985).

5 Hovis, J. S. \& Boxer, S. G. Patterning barriers to lateral diffusion in supported lipid bilayer membranes by blotting and stamping. Langmuir 16, 894-897, (2000).

6 Subramaniam, A. B., Lecuyer, S., Ramamurthi, K. S., Losick, R. \& Stone, H. A. Particle/Fluid Interface Replication as a Means of Producing Topographically Patterned Polydimethylsiloxane Surfaces for Deposition of Lipid Bilayers. Adv. Mater. 22, 2142-+, (2010).

7 Adamson, A. W. \& Gast, A. P. Physical Chemistry of Surfaces. 6 edn, (Wiley Inter-Science, 1997).

8 Nédélec, F., Surrey, T. \& Maggs, A. C. Dynamic Concentration of Motors in Microtubule Arrays. Physical Review Letters 86, 3192-3195, (2001).

9 Johnson, D. A., Voet, J. G. \& Taylor, P. Fluorescence energy transfer between cobra alpha toxin molecules bound to the acetylcholine receptor. Journal of Biological Chemistry 259, 5717-5725, (1984).

10 Kahya, N., Scherfeld, D., Bacia, K., Poolman, B. \& Schwille, P. Probing lipid mobility of raft-exhibiting model membranes by fluorescence correlation spectroscopy. Journal of Biological Chemistry 278, 28109-28115, (2003).

11 Shaw, J. E. et al. Correlated fluorescence-atomic force microscopy of membrane domains: Structure of fluorescence probes determines lipid localization. Biophysical Journal 90, 2170-2178, (2006).

12 Axelrod, D., Koppel, D. E., Schlessinger, J., Elson, E. \& Webb, W. W. Mobility measurement by analysis of fluorescence photobleaching recovey kinetics. Biophysical Journal 16, 1055-1069, (1976). 
13 Yoon, T.-Y. et al. Topographic control of lipid-raft reconstitution in model membranes. Nature Materials 5, 281-285, (2006).

14 Magonov, S. N., Elings, V. \& Whangbo, M. H. Phase imaging and stiffness in tapping-mode atomic force microscopy. Surface Science $\mathbf{3 7 5}$, L385-L391, (1997).

15 Yoon, T.-Y. et al. Topographic control of lipid-raft reconstitution in model membranes. Nat Mater 5, 281-285, (2006).

16 Goksu, E. I. et al. Effect of Support Corrugation on Silica XerogelSupported Phase-Separated Lipid Bilayers. Langmuir 25, 3713-3717, (2009).

17 Parthasarathy, R., Yu, C.-h. \& Groves, J. T. Curvature-Modulated Phase Separation in Lipid Bilayer Membranes. Langmuir 22, 5095-5099, (2006). 